\title{
Understanding the origin of the positron annihilation line and the physics of supernova explosions
}

\author{
F. Frontera ${ }^{1,2,3}$ (D) . E. Virgilli ${ }^{1}$. C. Guidorzi ${ }^{1} \cdot$ P. Rosati ${ }^{1}$ - R. Diehl ${ }^{4}$. T. Siegert ${ }^{5}$. \\ C. Fryer ${ }^{6}$ - L. Amati ${ }^{2}$ - N. Auricchio ${ }^{2}$. R. Campana ${ }^{2}$ - E. Caroli ${ }^{2}$ - F. Fuschino ${ }^{2}$. \\ C. Labanti ${ }^{2}$ - M. Orlandini ${ }^{2}$ - E. Pian ${ }^{2}$ - J. B. Stephen ${ }^{2}$ - S. Del Sordo ${ }^{7}$. \\ C. Budtz-Jorgensen ${ }^{8} \cdot$ I. Kuvvetli ${ }^{8}$. S. Brandt ${ }^{8}$. R. M. Curado da Silva9,10 . \\ P. Laurent ${ }^{11} \cdot$ E. Bozzo ${ }^{12}$. P. Mazzali ${ }^{13} \cdot$ M. Della Valle ${ }^{3,14}$
}

Received: 18 July 2020 / Accepted: 2 March 2021 / Published online: 22 June 2021

(C) The Author(s) 2021

\begin{abstract}
Nuclear astrophysics, and particularly nuclear emission line diagnostics from a variety of cosmic sites, has remained one of the least developed fields in experimental astronomy, despite its central role in addressing a number of outstanding questions in modern astrophysics. Radioactive isotopes are co-produced with stable isotopes in the fusion reactions of nucleosynthesis in supernova explosions and other violent events, such as neutron star mergers. The origin of the $511 \mathrm{keV}$ positron annihilation line observed in the direction of the Galactic Center is a 50-year-long mystery. In fact, we still do not understand whether its diffuse large-scale emission is entirely due to a population of discrete sources, which are unresolved with current poor angular resolution instruments at these energies, or whether dark matter annihilation could contribute to it. From the results obtained in the pioneering decades of this experimentally-challenging window, it has become clear that some of the most pressing issues in high-energy astrophysics and astro-particle physics would greatly benefit from significant progress in the observational capabilities in the keV-to-MeV energy band. Current instrumentation is in fact not sensitive enough to detect radioactive and annihilation lines from a wide variety of phenomena in our and nearby galaxies, let alone study the spatial distribution of their emission. In this White Paper (WP), we discuss how unprecedented studies in this field will become possible with a new low-energy gamma-ray space experiment, called ASTENA (Advanced Surveyor of Transient Events and Nuclear Astrophysics), which combines new imaging, spectroscopic and polarization capabilities. In a separate WP (Guidorzi et al. 2021), we discuss how the same mission concept will enable new groundbreaking studies of the
\end{abstract}

F. Frontera

frontera@fe.infn.it

Extended author information available on the last page of the article. 
physics of Gamma-Ray Bursts and other high-energy transient phenomena over the next decades.

Keywords $\mathrm{X}-/ \gamma$-ray telescopes - Space mission concept · ESA voyage 2050 . Origin of positron annihilation line from Galactic bulge region · Dark matter from the Galactic Center region · Nucleosynthesis study in novae · type I and core-collapse supernovae $\cdot$ Physical origin of the Phillips law $\cdot$ Nuclear line distribution across supernova remnants

\section{Introduction}

Nuclear science is key to understanding the energy that makes stars and supernovae shine, as well as the synthesis of the variety of chemical elements that evolved from the H-He mix left behind after the big bang. Despite its central role however, nuclear astrophysics has mostly remained a theoretical field, rather than an experimentallyconfirmed science. It is in fact one of the most challenging and least developed fields of astronomy. The limited sensitivity and imaging capabilities of the gamma-ray instrumentation flown thus far has limited nuclear astrophysics studies only to the brightest nearby sources. This suggests that a large discovery space could be opened in this field by expanding the experimental frontier. Among the most relevant open issues of astro-particle physics today is certainly the origin of the $511 \mathrm{keV}$ positron annihilation line from the Galactic bulge region and whether the seemingly diffuse emission is made up of a population of discrete unresolved sources. Another longoutstanding issue is a detailed understanding of the physical processes that shape the explosion of Type-Ia and core-collapse supernovae, which has been hindered because of the poorly-developed state of nuclear astrophysics. Specifically, Type-Ia SN are the most important distance indicators on cosmological scales, thanks to an empirical relation (the Phillips law, [61]), that links their luminosity at maximum with the post-peak decline rate, thus making SN-Ia good standard candles. A landmark experimental advance in this field would make nuclear line diagnostics an essential tool to develop self-consistent physical models of SN-Ia light curves, which will lead to understand the nature of the current observed scatter and their behaviour as standard candles. More generally the exponential fading exhibited by SN light curves is an indirect evidence of elements being made [23]. Nuclear gamma-ray astronomy carried out with the proposed mission concept ASTENA would provide a direct experimental confirmation of explosive nucleosynthesis theory, both in the continuum and in the lines emitted following the decay of ${ }^{56} \mathrm{Co},{ }^{56} \mathrm{Ni},{ }^{44} \mathrm{Ti}$ and ${ }^{56} \mathrm{Fe}$.

\section{The $511 \mathrm{keV}$ positron annihilation line from the Galactic Center region}

A recollection of the discovery and early observations of the $511 \mathrm{keV}$ line can be found in the historical review on hard $\mathrm{X}-$ /soft gamma-ray astronomy by [17]. For 
a recent review including the astrophysical implications, see [62]. The two fundamental questions are: 'Where do the positrons come from?' and 'Why does the emission appear so different to our other views of our Galaxy?' - this is generally known as 'the positron line puzzle'. In the following, we will provide a short overview of the findings over the last 50 years and describe the potential of, and need for, a sub-arcminute telescope to solve the controversy of the $511 \mathrm{keV}$ emission.

\subsection{Emission morphology - Diffuse or not?}

The $511 \mathrm{keV}$ emission was discovered by [45] in a balloon flight in 1970, refined by [52], and confirmed in another flight in 1979 [53]. During the first 25 years of study, the line flux was found to be variable and sometimes undetected. In the late 1980s, the Solar Maximum Mission (SMM) consistently detected the $511 \mathrm{keV}$ line at a flux level of $2.3 \times 10^{-3}$ photons $\mathrm{cm}^{-2} \mathrm{~s}^{-1}$ [66], comparable to the upper limits of some balloon flights earlier in the decade [54], and with other balloons flights later (e.g. GRIS, [33]). With the satellite experiment OSSE on CGRO [63], the line flux was found stable at a flux level of $\sim 10^{-3}$ photons $\mathrm{cm}^{-2} \mathrm{~s}^{-1}$, and the apparent variability was attributed to peculiar diffuse emission which was only partly captured by the different fields of view of previous instruments [2, 55]. This earliest mapping of the $511 \mathrm{keV}$ line emission was performed by [64] using CGRO/OSSE, WIND/TGRS [74], and SMM data. Unlike at other wavelengths, the emission was dominated by a central bulge, a probably truncated emission from the Galactic plane, and an enhancement at positive latitudes above the Galactic Center (GC). With INTEGRAL SPI, the morphology became clearer over time, with a significant detection of the bulge after 1.5 years [47], a possible asymmetric disc emission after 3 years [81], and the correction of these findings after 8 years [13], describing the $511 \mathrm{keV}$ map with a shifted bulge and a low surface-brightness disc. With more than 10 years of INTEGRAL SPI data, spectral positron annihilation features are detected with $\sim 70 \sigma$ in the bulge and $\sim 12 \sigma$ in the disc [69], with an angular resolution of $\sim 3^{\circ}$. While OSSE's 'Galactic Positron Fountain' has never been confirmed, a new debate on whether the disc is actually thin or shows a large scale height is ongoing $[69,73]$. The bulge emission is now found to be highly symmetric and can be described by a combination of two 2D Gaussians with a slight shift towards negative longitudes [73], including a dominating component of $\sim 6 \mathrm{deg}$ (FWHM), and a possible point source at the GC with a flux of $(0.8 \pm 0.2) \times$ $10^{-4}$ photons $\mathrm{cm}^{-2} \mathrm{~s}^{-1}$. The most recent flux estimate for the entire Milky Way is $(2.7 \pm 0.3) \times 10^{-3}$ photons $\mathrm{cm}^{-2} \mathrm{~s}^{-1}$, which converts to an annihilation rate (luminosity) of $\sim 5 \times 10^{43} \mathrm{e}^{+} \mathrm{s}^{-1}$ [69]. This number, if not steadily decaying from a much larger supply, must be sustained by one or more sources, which to date, are still unknown ${ }^{1}$.

\footnotetext{
${ }^{1} \mathrm{The} \beta^{+}$-decay of ${ }^{26} \mathrm{Al}$ is believed to account for about $10 \%$ of the total flux, but a 'smoking gun' evidence for this candidate source has not yet been found.
} 


\subsection{Candidate sources - Detecting sources in flagranti}

Due to the seemingly asymmetric disc reported by [81], an attempt was made to correlate the emission with the population of Low Mass X-ray Binaries (LMXRBs), dominating at $\gtrsim 10 \mathrm{keV}$, as the distribution of the 71 known LMXRBs appeared tantalizingly similarly asymmetric (45 at negative longitudes, 26 at positive longitudes). While the asymmetry was not confirmed (see Fig. 1, from [81] and [13]), LMXRBs may still have a large contribution to the positron content of the Milky Way. As a result of annihilation of positrons produced in $\mathrm{e}^{+}-\mathrm{e}^{-}$pair-dominated jets [18] of black-hole systems or of $\gamma-\gamma$ interactions in the inner regions of hightemperature/high-density accretion discs of XRB systems, the population of XRBs can potentially account for all the positrons seen to annihilate. This microquasar$511 \mathrm{keV}$ conjecture is supported by detections of thermal pair plasma emission from three sources in outburst: 1E 1740.7-2942, also known as "the Great Annihilator" [12], Nova Musca [34], and V404 Cygni [68] - all of which have been debated though. Also, given the thousands of Chandra sources revealed within only $17 \times 17$ arcmin around Sgr A* [40], the possibility that the $511 \mathrm{keV}$ line is due to a superposition of discrete sources remains an attractive solution.

Sgr $A^{*}$ itself is also a prominent candidate source in this context, either by a pairdominated jet, 'calming down' from past AGN activity, or triggering a starburst event several million years ago $[3,62]$. The possibility of a point-like source in the GC $[69,73]$ requires confirmation, and still opens intriguing possibilities concerning the positron puzzle. Given the low angular resolution $\left(2.7^{\circ}\right)$ of SPI [76] and other experiments, [6] recall that "from the data it is unclear whether the emission is truly diffuse, or if it originates either from a single discrete source (e.g. SgrA* or 1E 1740.7-2942 [...]) or from a small number of discrete but unresolved sources.". Within $2.7^{\circ}$ at a distance of $8.1 \mathrm{kpc}$, a region as large as $400 \mathrm{pc}$ fits into only one PSF of SPI. This size is reminiscent of the Central Molecular Zone, harbouring not only XRBs, but also molecular gas in which positrons may prefer to annihilate, as well as a dense cluster of stars - the nuclear stellar cluster. The population of flaring M- to G-type stars may in fact provide a complete solution as well: [8] estimated that the $\sim 10^{11}$ stars in the Milky Way show a quasi-persistent $511 \mathrm{keV}$ flux from intermittent stellar flares,
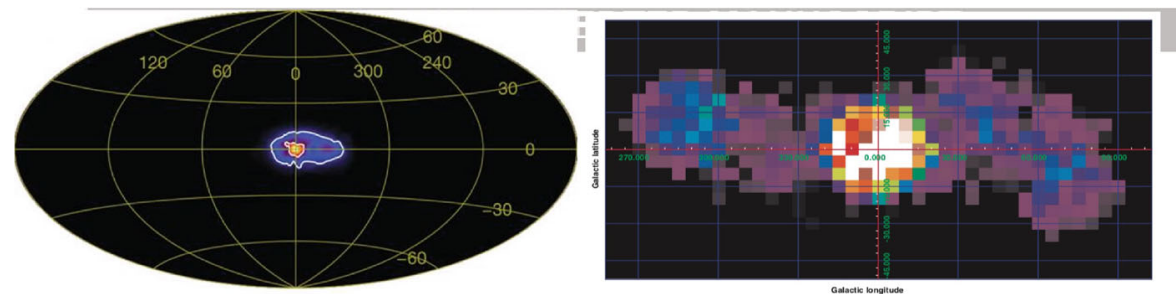

Fig. 1 Sky map of the $511 \mathrm{keV}$ positron annihilation line found with the INTEGRAL SPI telescope by [81] (left panel) and by [13] (right panel). According to [81], the integrated fluxes in the negative $(-50<$ $l<0)$ and positive $(0<l<50)$ longitude bands, within $10 \mathrm{deg}$ of the Galactic plane, are $(4.3 \pm 0.5) \times$ $10^{-3}$ photons $\mathrm{cm}^{-2} \mathrm{~s}^{-1}$ and $(2.4 \pm 0.5) \times 10^{-3}$ photons $\mathrm{cm}^{-2} \mathrm{~s}^{-1}$, respectively. The integrated flux found by [13] was consistent with that reported by [81], but no asymmetry was found 
producing pions and $\beta^{+}$-unstable nuclei. The sheer number of stars may appear as a diffuse flux of the order of $10^{-3}$ photons $\mathrm{cm}^{-2} \mathrm{~s}^{-1}$ at $511 \mathrm{keV}$ and would readily explain the bulge emission. The nuclear stellar cluster as well as a large number of the Milky Way's globular clusters would stand out of the otherwise diffuse glow and provide direct evidence for these types of scenarios. With arcmin resolution, it would furthermore be possible to distinguish among mildly relativistic, quiescent XRB outflows [7] or stellar flares as the latter would directly follow the globular cluster's radial profile.

A possible explanation for the positron puzzle is the $\beta^{+}$-decay from radioactive ejecta of massive stars and their core-collapse supernovae (CCSNe), as well as of white dwarf systems in classical novae $(\mathrm{CNe})$ or thermonuclear type Ia supernovae (SNe Ia). Here, the most important nuclei are ${ }^{26} \mathrm{Al}\left(T_{1 / 2} \approx 717 \mathrm{kyr} ; 1809 \mathrm{keV}\right.$ line; massive star winds, CCSNe, CNe), ${ }^{44} \mathrm{Ti}\left(T_{1 / 2} \approx 60 \mathrm{yr} ; 68,78,1157 \mathrm{keV}\right.$ lines; $\mathrm{CCSNe}$, SNe Ia, e.g. [72]), ${ }^{22} \mathrm{Na}\left(T_{1 / 2} \approx 2.75 \mathrm{yr} ; 1275 \mathrm{keV}\right.$ line; $\left.\mathrm{CNe}\right)$, and ${ }^{56} \mathrm{Co}$ $\left(T_{1 / 2} \approx 0.2 \mathrm{yr} ; 847,1238 \mathrm{keV}\right.$ lines, ${ }^{2} \mathrm{CCSNe}$, SNe Ia, e.g. [22]). While the latter is the main energy producer for the light curves of SNe Ia and consequently the lineof-sight escape from the main sources is very uncertain (e.g. [59]), all the decay positrons have in common that they first have to slow down to explain the measured signal: $\beta^{+}$-decay typically emits $\lesssim \mathrm{MeV}$ positrons, but the line shape suggests positrons at $\mathrm{eV}$ energies (e.g. [20, 44, 69, 71]). This slowing down is connected to the propagation of cosmic-ray positrons, and the interplay of the environmental conditions near the initial source and the properties of the interstellar medium between source and region of annihilation. This becomes even more important when pairproduced positrons from interactions of high-energy photons in the strong magnetic fields of pulsars are considered (e.g. [25]). In addition to these away-from-the-source scenarios, CNe systems are expected to show a short $\sim 1 \mathrm{hr}$ flash or $511 \mathrm{keV}$ radiation from the decay of short-lived ${ }^{13} \mathrm{~N}$ and ${ }^{18} \mathrm{~F}$, which however has never been detected. In order to distinguish between either of these scenarios, or to establish whether more sources are required to explain the total signal, superb line sensitivity as well as unprecedented angular resolution is required. The focusing telescope part of the ASTENA mission concept we are proposing here will have a $511 \mathrm{keV}$ sensitivity of $\sim 7 \times 10^{-6}$ in $1 \mathrm{Ms}$ (see Fig. 17), with which it will be possible: 1 ) to detect $511 \mathrm{keV}$ emission from SNe Ia up to a distance of $\sim 10 \mathrm{Mpc}$ to directly measure the escape fraction of positrons, 2) to see $\mathrm{CNe}$ flashes up to $5 \mathrm{kpc}$ and detect the decay positrons from ${ }^{22} \mathrm{Na}$ up to $1 \mathrm{kpc}, 3$ ) to unravel the annihilation regions in the vicinity of massive stars (e.g. Cygnus OB associations), 4) to discover the late light curve heating of CCSNe by measuring positron annihilation from ${ }^{44} \mathrm{Ti}$ in Cassiopeia $\mathrm{A}$, and 5) to possibly detect extragalactic emission regions of $511 \mathrm{keV}$ in the LMC and M31.

Ultimately, with our proposed instrumentation one will be able to discriminate between the diffuse vs. discrete origin of the $511 \mathrm{keV}$ line, by performing a scan of selected regions near the GC, with a telescope with a much higher sensitivity and angular resolution than any current or planned instrumentation.

\footnotetext{
${ }^{2}$ From the decay of ${ }^{56} \mathrm{Ni}\left(T_{1 / 2} \approx 6 \mathrm{~d} ; 158,812 \mathrm{keV}\right.$ lines $)$.
} 


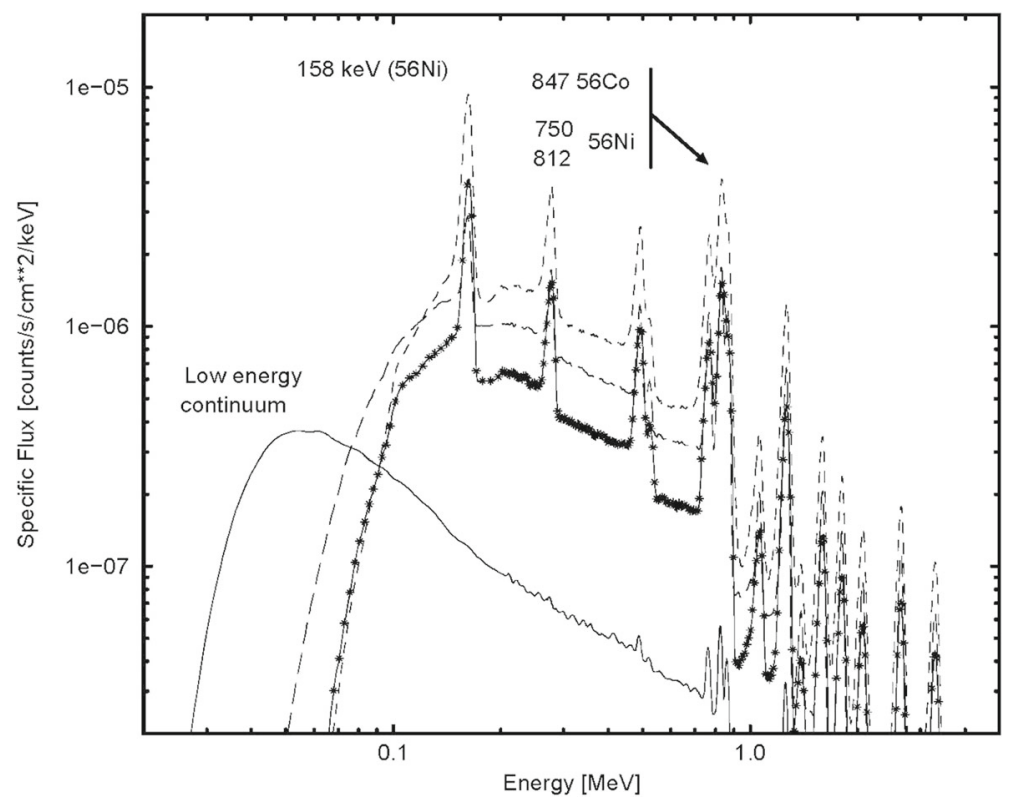

Fig. 2 Expected gamma-ray spectrum of a SN Ia at 20 days from the explosion. The different curves refer to different possible explosion mechanisms (deflagration, detonation and delayed detonation). Reprinted from [35]

\subsection{The $\mathbf{5 1 1} \mathrm{keV}$ line and the quest for dark matter}

As summarised above, the pure astrophysical explanation of the $511 \mathrm{keV}$ diffuse emission extending over $\sim 10 \mathrm{deg}^{2}$ around the GC is far from being clear, and it remains a challenge to explain the spectrum, intensity and morphology of the INTEGRAL signal with candidate sources (see [62] for a review). Therefore, since the first detailed map of the GC by INTEGRAL SPI was released (Fig. 1), many studies have tried to establish whether annihilation or decay of dark matter (DM) particles can possibly contribute to the $511 \mathrm{keV}$ line production (e.g. [10, 29], see also [9]). Even though the central slope of the dark matter density profile of our galaxy is still not well known, the number density of annihilating positrons depends on the square of the DM number density, which necessarily peaks near the GC. These first and more recent studies (e.g. [19]) have constrained the possible annihilation channels, annihilation cross sections, and dark matter mass range, finding that specific models with MeV-to-GeV masses can reproduce the $511 \mathrm{keV}$ signal, and at the same time being consistent with the relic density of DM from cosmological observations. Other studies have instead found $\mathrm{MeV}$ annihilating relic dark matter inconsistent with cosmological data ([83]). It is clear that the lack of knowledge of the physics of DM, makes these predictions quite speculative, especially when the contribution of discrete, currently unresolved sources to the $511 \mathrm{keV}$ extended signal, is still not known. By disentangling the point source contribution from the $511 \mathrm{keV}$ emission 


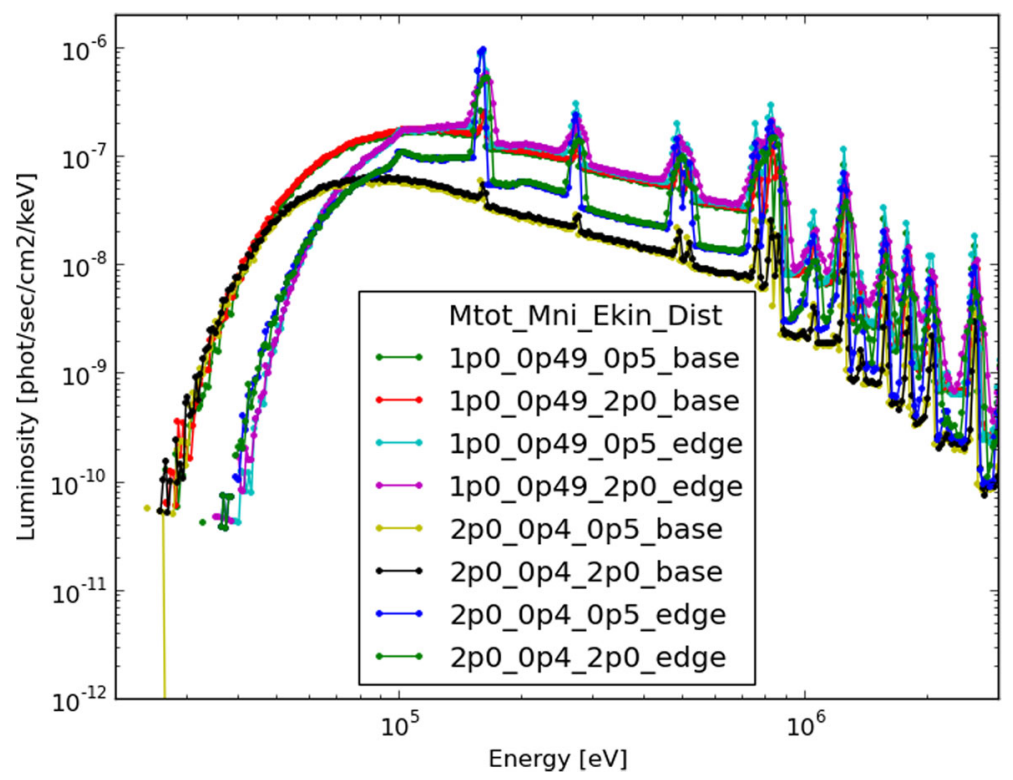

Fig. 3 Expected gamma-ray spectrum of a SN Ia at 20 days from the explosion for a range of models where the explosion energy, total mass, nickel mass and its distribution are varied across the range of current explosion models [42]

with highly sensitive, sub-arcminute resolution maps, one will obtain a firm measurement of a possible excess of positrons in the galactic bulge. This will in turn significantly narrow down the range of DM physical parameters consistent with such excess, or lack thereof.

\section{Line emission from radioactive nuclei produced in supernova explosions of types la and core-collapse}

\subsection{Type la SNe}

SN Ia are the outcome of the thermonuclear explosion of either a single Carbon/Oxygen white dwarf with a mass near the Chandrasekhar limit in a close binary system or a pair of merging white dwarfs. The burning of the Carbon/Oxygen mixture provides the required kinetic energy, while the decay of ${ }^{56} \mathrm{Ni} \longrightarrow{ }^{56} \mathrm{Co}$ with a half-life of 6.1 days and that of ${ }^{56} \mathrm{Co} \longrightarrow{ }^{56} \mathrm{Fe}$, with a half-life of 77.7 days, power the optical light curve, as shown by the SN2014J observations with INTEGRAL, 50 and 100 days after the explosion [21,27], in agreement with theoretical expectations, e.g., by [49]. Using the SPI and the IBIS/ISGRI telescopes aboard INTEGRAL, [21] reported the detection of the ${ }^{56} \mathrm{Co}$ lines at energies of $847 \mathrm{keV}$ and $1238 \mathrm{keV}$ and a $\gamma$-ray continuum in the $200-400 \mathrm{keV}$ band. Detection of the ${ }^{56} \mathrm{Co}$ gamma rays establishes directly that $0.6 \pm 0.1$ solar masses of radioactive ${ }^{56} \mathrm{Ni}$ were synthesized 


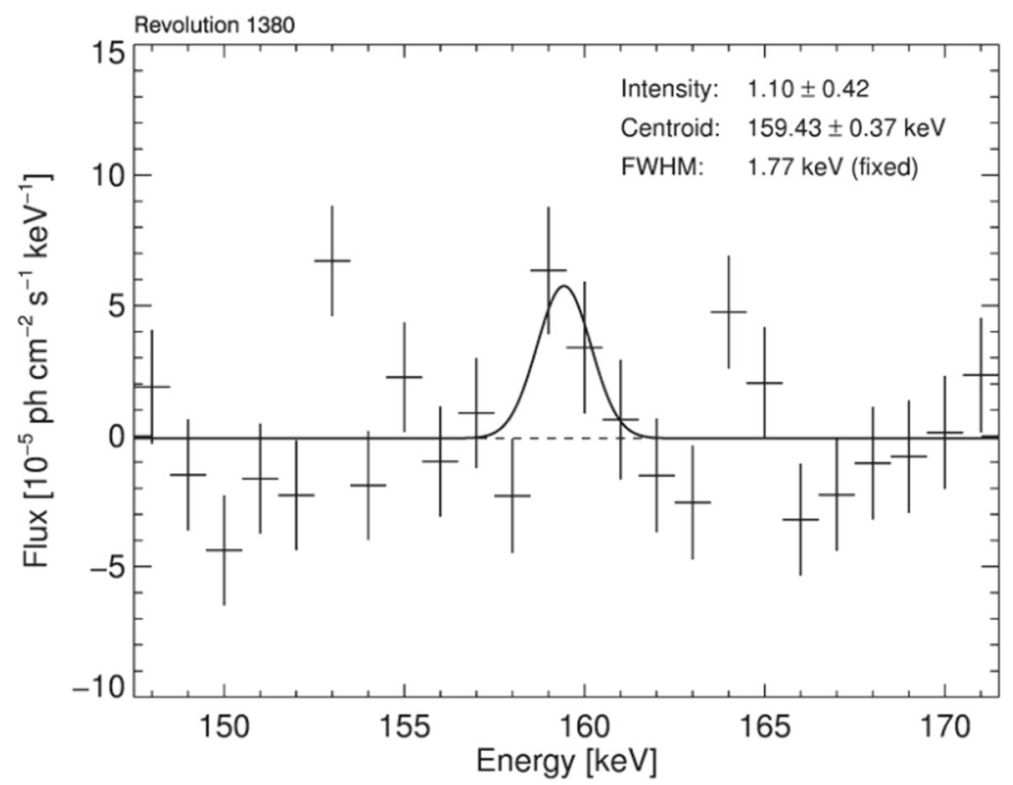

Fig. 4 The $158 \mathrm{keV}$ line due to the ${ }^{56} \mathrm{Ni}$ decay of SN2014J, as observed with $150.24 \mathrm{ks}$ INTEGRAL SPI observations, 3 weeks after the explosion. The line intensity is given in $10^{-4}$ photons cm $\mathrm{cm}^{-1}$ units. Reprinted from [28]

during the explosion [21, 27]. On the other side, about three weeks after the explosion of this same SN, from data obtained with the SPI telescope, [28] reported the detection of both the 158 and $812 \mathrm{keV}$ lines from the ${ }^{56} \mathrm{Ni}$ decay, with intensities of

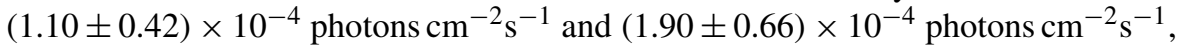
respectively. This evidence of radioactive ${ }^{56} \mathrm{Ni}$ near the surface, later confirmed by [43], points to explosion asymmetries, if not an initial, triggering, surface explosion (see discussion in [28]).

Understanding the physical processes leading to explosions of type Ia SNe is very important, given that SN Ia are used as distance indicators at cosmological scale, extrapolating the empirical Phillips correlation far beyond the established nearby redshift regime. The Phillips relation between maximum luminosity and post-peak decline rate allows one to standardize the absolute luminosity and correct for individual SN Ia properties, as shown by reducing the light peak dispersion of the observed SNe from $1.5 \mathrm{mag}$ to $0.1-0.2 \mathrm{mag}$. A robust physical modeling of the Phillips law could further improve the accuracy in the distance measurement, as, e.g., effects of different metallicity on the explosion would be included in a truly-physical model. Given the primary role of ${ }^{56} \mathrm{Ni}$ and its products in powering the light curve, the measurement of SNe Ia gamma-ray lines and continuum spectrum is a crucial diagnostic tool to understand the physics of the explosion and its evolution [35].

At early times from the explosion (see Fig. 2), the spectrum is dominated by the ${ }^{56} \mathrm{Ni}$ main lines (158 keV and $812 \mathrm{keV}$ ) and from the $847 \mathrm{keV}$ and $1238 \mathrm{keV}$ lines due to the radioactive ${ }^{56} \mathrm{Co}$. The strongest lines are the $158 \mathrm{keV}$ line and the 812 and 
$847 \mathrm{keV}$ lines. However, given the limited energy resolution of gamma-ray detectors, it is difficult to separate the last two lines. Thus, for the study of the early evolution of the SNe Ia, the study of the $158 \mathrm{keV}$ offers the best approach.

The strength of the gamma-ray spectra depends on the mass and velocities of the ejecta, nickel mass, and distribution. Figure 3 shows the dependence of gamma-ray lines on these explosion properties. A direct access to the total flux and line features will provide a novel powerful tool to measure these physical properties, thus probing the nature of the thermonuclear engine behind these supernovae.

In Fig. 4, we show the $158 \mathrm{keV}$ line detected with INTEGRAL from SN2014J, with an exposure time of $150 \mathrm{ks}$ [28]. The estimated line intensity of $(1.1 \pm 0.4) \times 10^{-4}$ photons $\mathrm{cm}^{-2} \mathrm{~s}^{-1}$ corresponds to a detection confidence level of $2.5 \sigma$. For comparison, the ASTENA Narrow Field Telescope (NFT) will reach a $3 \sigma$ sensitivity at the same energy (see Fig. 17) of approximately $10^{-6}$ photons $\mathrm{cm}^{-2} \mathrm{~s}^{-1}$ in $100 \mathrm{ksec}$ observations.

As a result, ASTENA NFT will be capable of detecting lines from SNe that synthesize lower masses of ${ }^{56} \mathrm{Ni}$ than those found near the surface of SN2014J $(0.06 \mathrm{M} \odot$, [28], $D=3.3 \mathrm{Mpc}$ ), or to detect lines from SN Ia at greater distances. Given the significantly higher sensitivity and angular resolution of ASTENA, we also expect to be able to study the spatial distribution and the evolution of both line and continuum in the case of Galactic SNe. From theoretical estimates (Figs. 2 and 3) as well as observational evidence (SN2014J), we expect to significantly increase the number of SNe Ia that could be investigated in gamma rays. ASTENA's superior line sensitivity around $158 \mathrm{keV}$ as well as the more than two orders of magnitude better continuum sensitivity around $200-400 \mathrm{keV}$ would allow to follow-up between 50 and 200 SNe Ia per year, up to a distance of $120 \mathrm{Mpc}(z \lesssim 0.03)$. Consequently, ASTENA would tremendously contribute to understanding the diversity of SNe Ia, their different explosion mechanisms (progenitor problem), as well as shed light on supernova cosmology.

\subsection{Core collapse $\mathrm{SNe}$}

In Core Collapse supernova explosions (CCSN; also called SN Ib/c or SN II) that result from collapses of massive stars at the end of their stellar evolution, one of the most diagnostic synthesized elements is ${ }^{44} \mathrm{Ti}$. It decays according to the chain ${ }^{44} \mathrm{Ti}$ $\longrightarrow{ }^{44} \mathrm{Sc} \longrightarrow{ }^{44} \mathrm{Ca}$ with a half life of $58.9 \pm 0.3$ yrs [1] and direct emission of lines at $4.1,67.9,78.4,511$, and $1,157 \mathrm{keV}$, with relative brightnesses, i.e., average numbers of photons per decay, of $17.4 \%, 87.7 \%, 94.7 \%, 188.8 \%$, and $99.9 \%$, respectively[36]. Given the long decay time, these lines are visible at late times once the explosions are entirely transparent. Of the above lines, those so far clearly detected are the $67.9 \mathrm{keV}, 78.4 \mathrm{keV}$, and $1157 \mathrm{keV}$ lines from Cas A, a SN that exploded in 1671, with BeppoSAX [77], INTEGRAL [65, 67] and NuSTAR [37], and from the Type II SN1987A [11, 36]. The integrated line intensity from Cas A is about $2 \times 10^{-5}$ photons $\mathrm{cm}^{-2} \mathrm{~s}^{-1}$. Thanks to the NUSTAR angular resolution (58 arcsec Half Power Diameter (HPD), [41]), it has been possible for the first time to study the spatial distribution of the line across the remnant [37, 38]. For SN1987A, whose distance is about $50 \mathrm{kpc}$, an estimate of a synthesized mass of ${ }^{44} \mathrm{Ti}$ of $(3.1 \pm 0.8) \times 10^{-4} \mathrm{M}_{\odot}$ 
was reported with INTEGRAL [36]; the NUSTAR measurement indicated that this intensity may have had a bias towards high values, the more-significant NUSTAR result is $(1.5 \pm 0.3) \times 10^{-4} \mathrm{M}_{\odot}$ [11]. Obtaining the yields for key metals detected in supernova remnants through X-rays depends sensitively on the excitation of the atoms and out-of-equilibrium effects lead to large errors in the total abundances derived from X-ray observations. Photons from radioactive decay do not suffer from these systematics, providing a more-robust abundance measurement. Because ${ }^{44} \mathrm{Ti}$ is produced in the innermost supernova ejecta, it is an ideal probe of the explosion mechanism and potential asymmetries. The NuSTAR results [37, 38] are considered to be the strongest evidence for an intrinsic explosion asymmetry and clumpiness, and the convective-engine paradigm behind core-collapse supernovae. ${ }^{44} \mathrm{Ti}$ production is extremely sensitive to the exact nature of the explosion (shock strengths and densities) and, by measuring the spatial distribution of the ${ }^{44} \mathrm{Ti}$ yields relative to iron, we can probe these diagnostic metal ratios.

The NFT telescope aboard ASTENA, thanks to its angular resolution (which is similar to that of NuSTAR), and its higher sensitivity, will be capable of performing more accurate spatial maps of the $67.9 \mathrm{keV}$ and $78.4 \mathrm{keV}$ lines across the remnant and extending this study to other remnants.

In addition, in the rare event of a nearby neutron star merger event or a Galactic supernova, a host of new isotopes may be detected [75]. For neutron star mergers within $10 \mathrm{Mpc}$, scientists can probe the mass and composition produced by heavy rprocess elements, firmly dictating the role that mergers play in r-process production and the robustness of their r-process composition [48, 84]. For Galactic supernovae, over 10 isotopes will be detected, some probing the stellar burning layers, others probing the explosion energy and mixing. These unique probes of cosmic explosions will dramatically improve our understanding of the progenitors and engines behind them.

\subsection{Classical nova systems}

$\mathrm{CNe}$ systems are expected (see $\$ 2.2$ ) on theoretical grounds to show a $\sim 1 \mathrm{hr}$ flash of $511 \mathrm{keV}$ radiation from the decay of short-lived ${ }^{13} \mathrm{~N}$ and ${ }^{18} \mathrm{~F}$. Given a rate of $\sim 14 \pm 3$ nova/yr for the galactic bulge [60] and the ASTENA sensitivity of $\sim 2 \times 10^{-5}$ at $511 \mathrm{keV}$ in $100 \mathrm{ks}$, it will be possible to detect CNe flashes up to $3 \mathrm{kpc}$ for a handful of Novae per year and to detect the decay positrons from ${ }^{22} \mathrm{Na}$ up to $\lesssim 1 \mathrm{kpc}$.

\section{ASTENA mission concept}

The ASTENA in-flight configuration is shown in Fig. 5, while its main properties are reported in Table 1. The instrumentation on board consists of a Wide Field MonitorImaging Spectrometer (WFM-IS) with a $2 \mathrm{keV}-20 \mathrm{MeV}$ passband, and a Narrow Field Telescope (NFT) with a 50-600 keV passband. The WFM-IS consists of an array of 12 units, two units on each side of the hexagon surrounding the NFT. All the units are offset by 15 degrees with respect to the axis of the NFT, as shown in Fig. 5. The NFT is a Laue lens telescope of about $3 \mathrm{~m}$ diameter and $20 \mathrm{~m}$ focal length. Part 


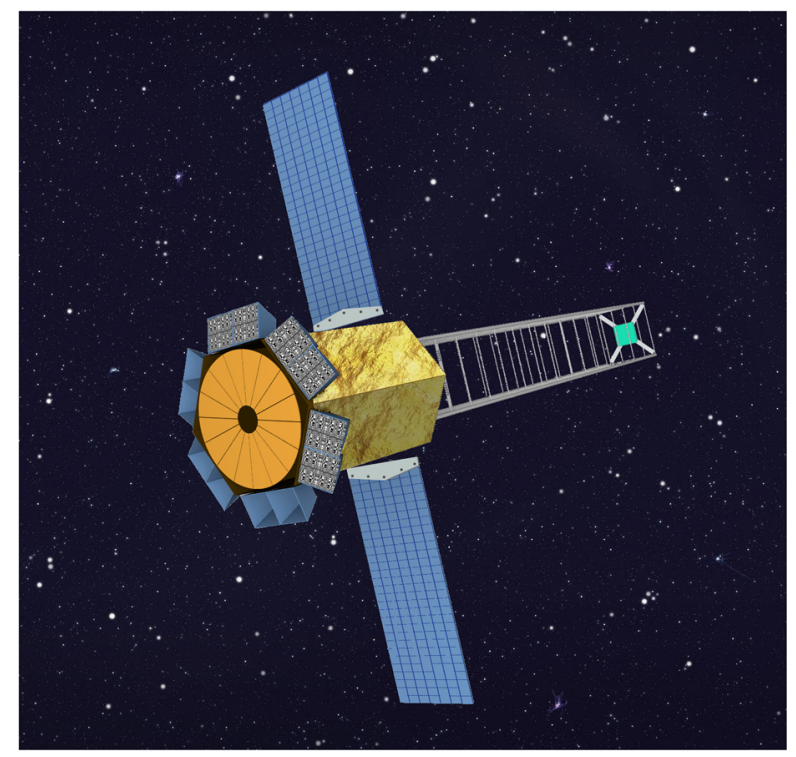

Fig. 5 Artistic view of ASTENA in-flight configuration

of the focal length $(5 \mathrm{~m})$ is inside the spacecraft and the remainder $(15 \mathrm{~m})$ is outside. The WFM-IS and the focal plane Position Sensitive Detector (PSD) are inside the spacecraft at launch (see left and central panels of Fig. 6). The ASTENA spacecraft can be accommodated inside the fairing of a Soyuz or Vega $C$ launcher (see right panel of Fig. 6).
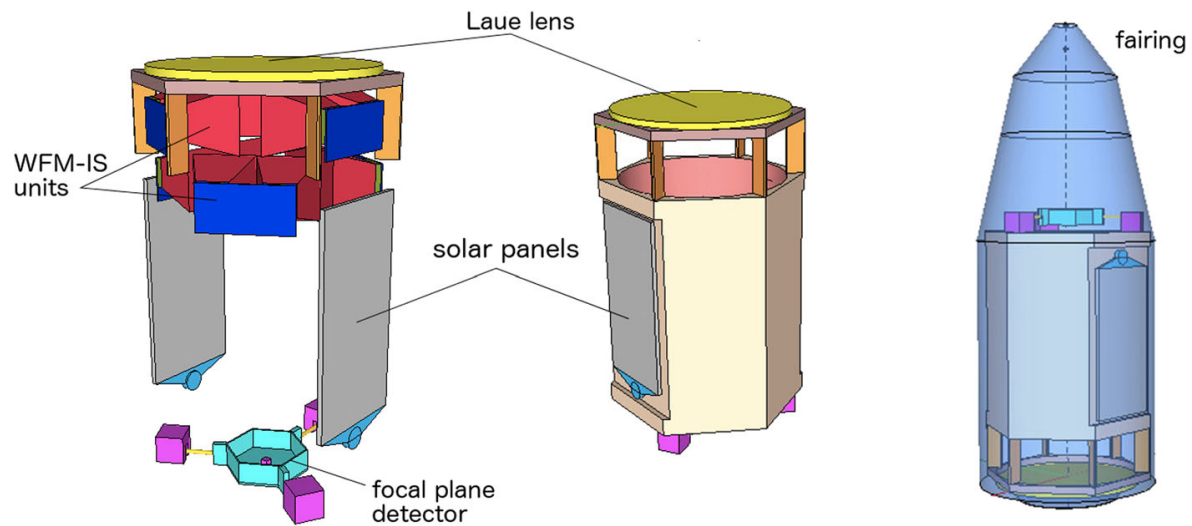

Fig. 6 Left panel: A view of the inner accommodation of the ASTENA payload before the launch. Central panel: A view of the inner side of the ASTENA spacecraft. Right panel: The ASTENA satellite in the fairing of the Soyuz launcher. Note that the fairing of the Vega C launcher can also host ASTENA 


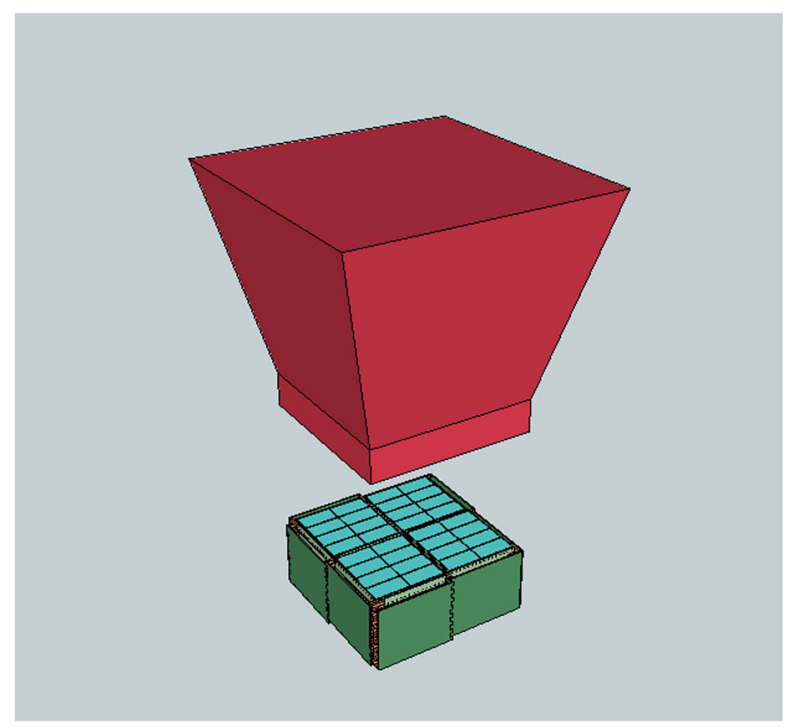

Fig. 7 Schematic view of a WFM-IS unit

\subsection{WFM-IS units}

Essentially, each detection unit (see Fig. 7) of the WFM-IS is a Position Sensitive Detector (PSD) surmounted by a coded mask at $70 \mathrm{~cm}$ distance. The mask is supported by 4 Aluminum slabs with, inside, a Tungsten layer about $500 \mu \mathrm{m}$ thick.
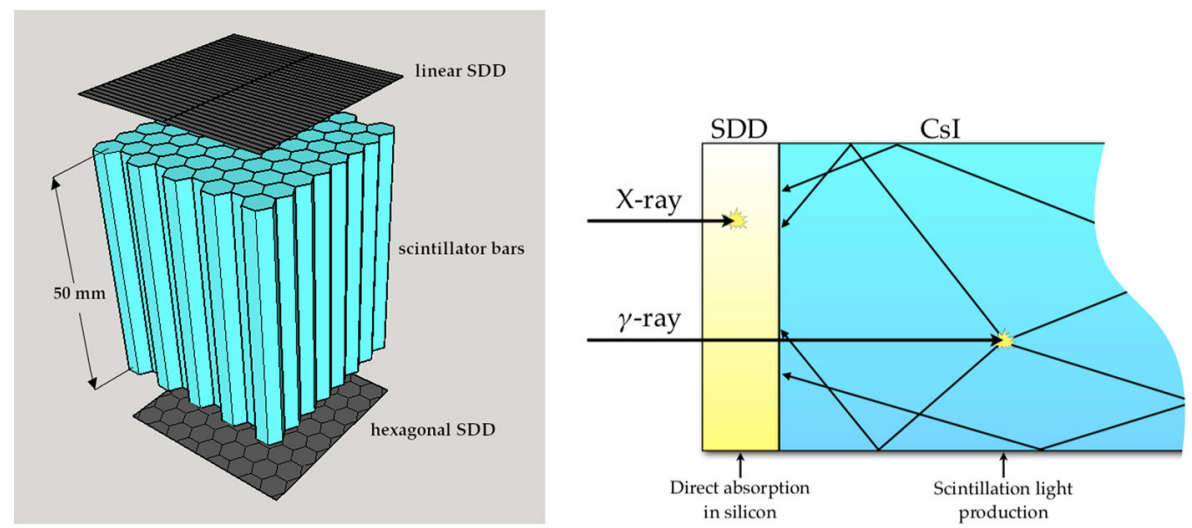

Fig. 8 Left: exploded view of a portion of a detection module of a WFM-IS unit. Right: working principle of a SDD coupled with a CsI scintillator crystal. The soft X-rays interact directly in the SDD while high energy photons pass through the SDD and are absorbed in the scintillator. Secondary photons are then detected with th SDD 

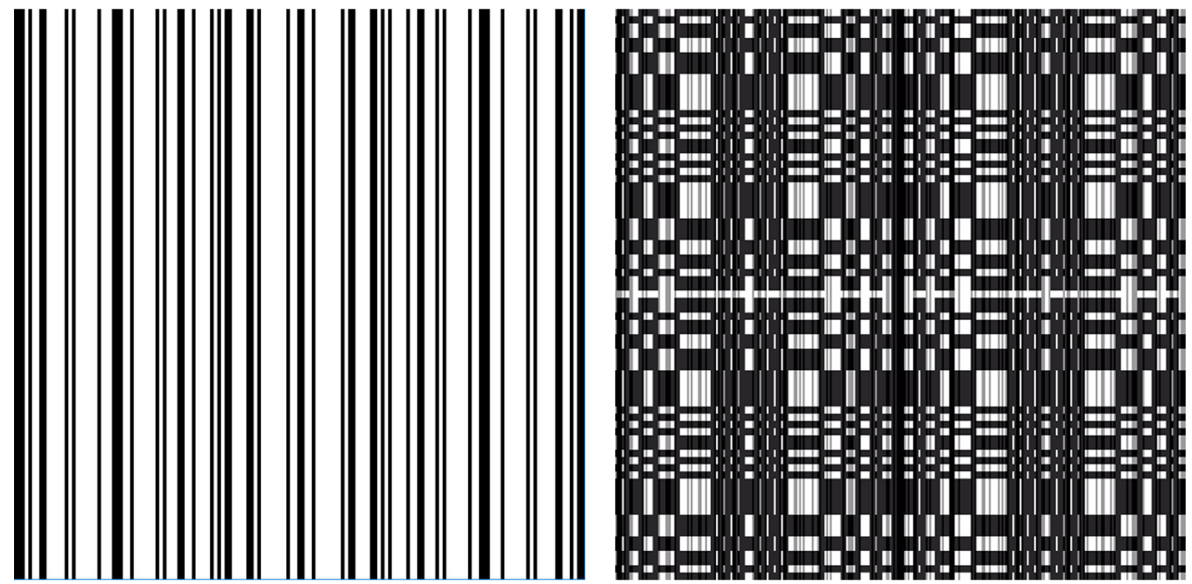

Fig. 9 Left: Top view of the 1-D low energy coded mask. Right: top view of the double scale coded mask

\subsubsection{The position sensitive detector unit}

The PSD unit consists of an array of $4 \times 8$ modules, each module consisting of 10 rows of hexagonal scintillator bars, with a distance between contiguous centres across hexagon flat sides of $5 \mathrm{~mm}$ (205 bars per module), read out, on the top (toward the

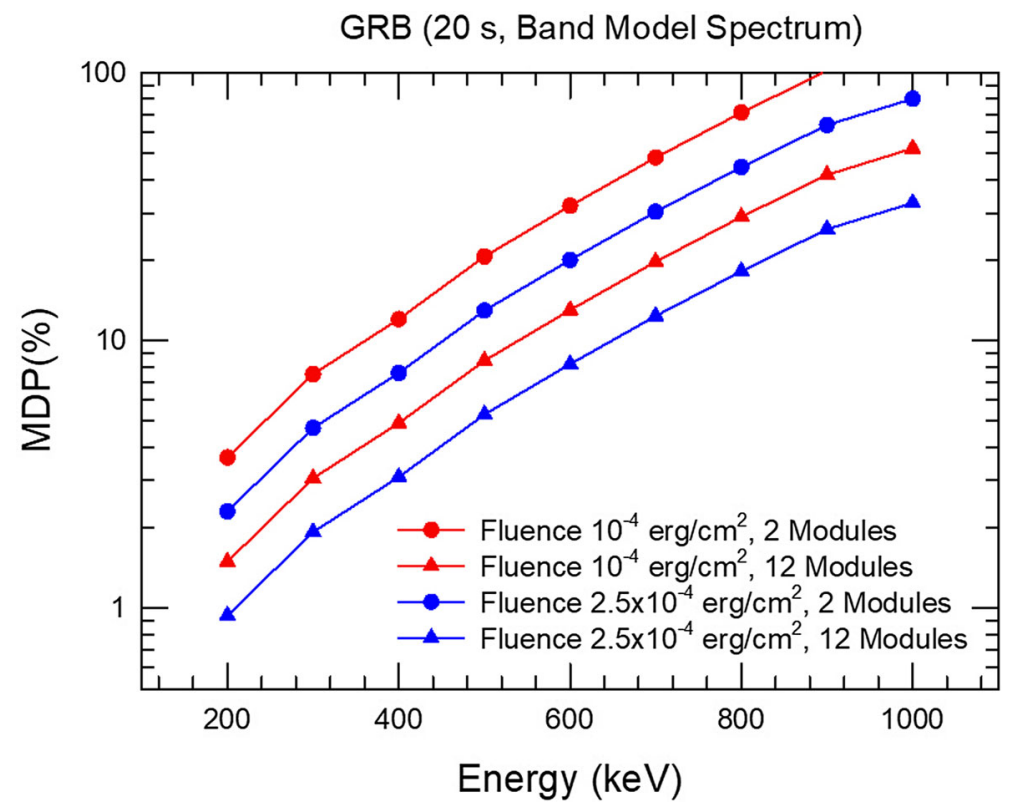

Fig. 10 Minimum Detectable Polarization at $3 \sigma$ level for a GRB of $20 \mathrm{~s}$ duration and 2 different fluences. The assumed spectrum is a Band law with low-energy photon index $\alpha=1.0$, high energy photon index $\beta=2.3$ and peak energy of the $E F(E)$ spectrum equal to $300 \mathrm{keV}$ 


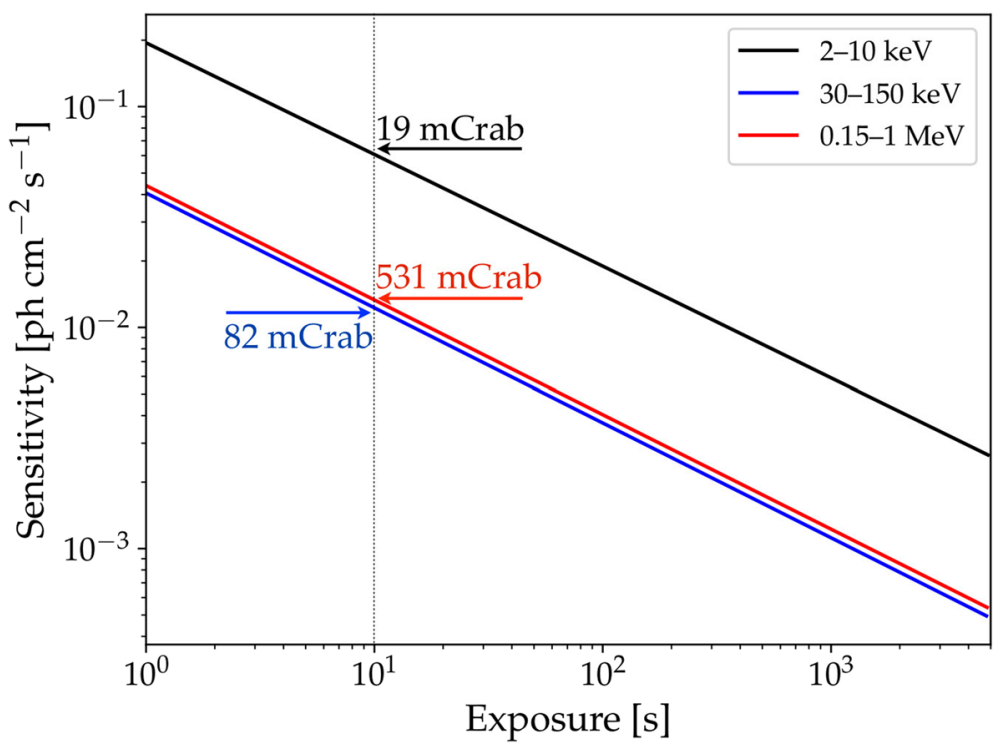

Fig. 11 Continuum sensitivity of the WFM-IS in three energy bands, in the case of a source in the FoV of all detection units

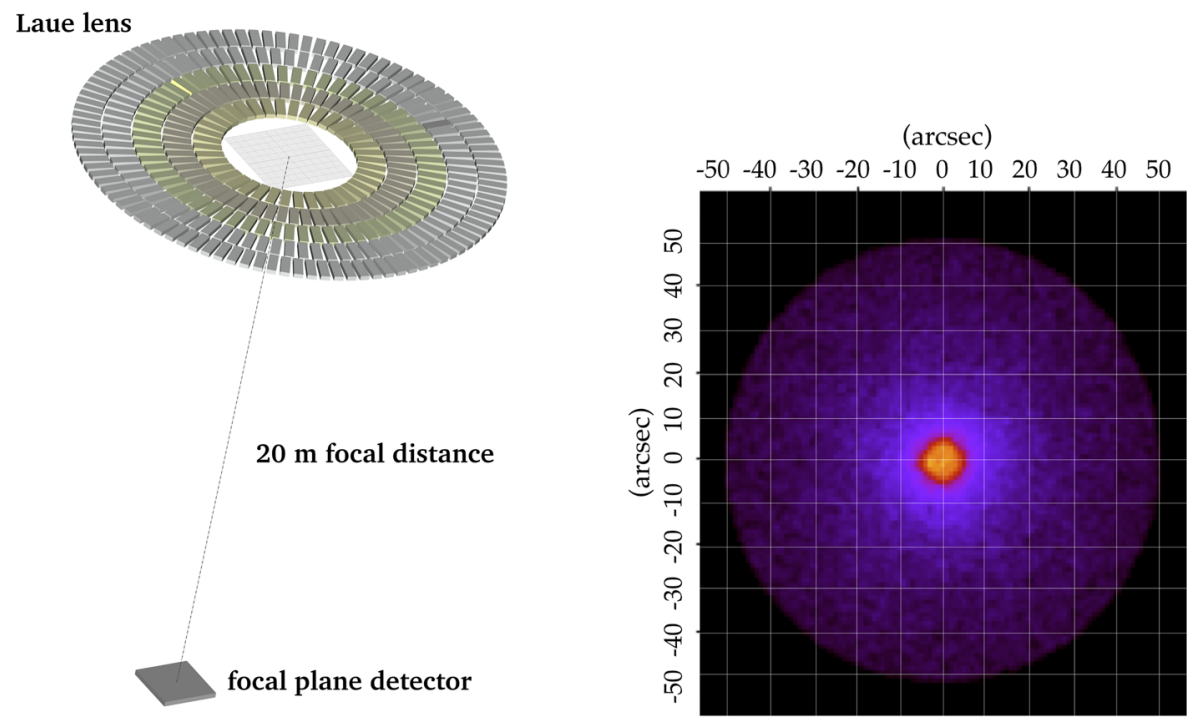

Fig. 12 Left: Sketch of the Laue lens adopted for the Narrow Field Telescope (NFT) which will be made of bent crystals. The detector and the Laue lens dimensions are not to scale. Right: Simulated two-dimensional PSF image achieved with the NFT, as obtained with our Laue lens physical model with diffractive bent crystals, in the case of an on-axis source 

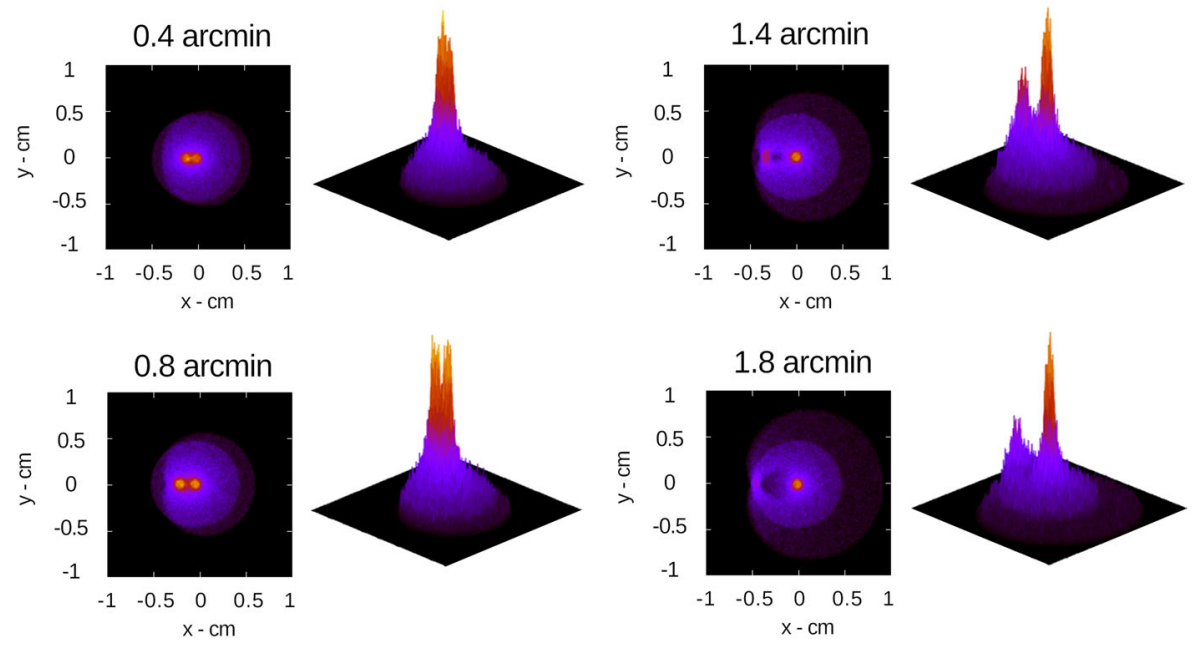

Fig. $132 \mathrm{D}$ and 3D PSF of the proposed lens in the case of two sources with different separations. One source is on-axis, while the other is located at increasing off-axis angles

mask), by linear multi-anode Silicon Drift Detectors (SDDs) $0.4 \mathrm{~mm}$ thick, and, on the bottom, by hexagonal single anode SDDs with a distance between centres across hexagon flat sides of $5 \mathrm{~mm}$ (see Fig. 8). The functioning principle of this detector is similar to that adopted for the X-Gamma-ray Imaging Spectrometer (XGIS) aboard the THESEUS mission [14]. It has the great advantage of a very broad passband (2 $\mathrm{keV}-20 \mathrm{MeV}$ ), a 3D position sensitivity to energy losses in the scintillator bars, and a very low intrinsic background, given its similarity to the phoswich system (see, e.g., [31]), in this case a "siswich" system as already demonstrated by [56, 57]. In the case of the WFM-IS units, the top SDDs have 4 linear anodes for each scintillator bar (see Fig. 8). This configuration provides a 1D position sensitivity of $1.25 \mathrm{~mm}$,

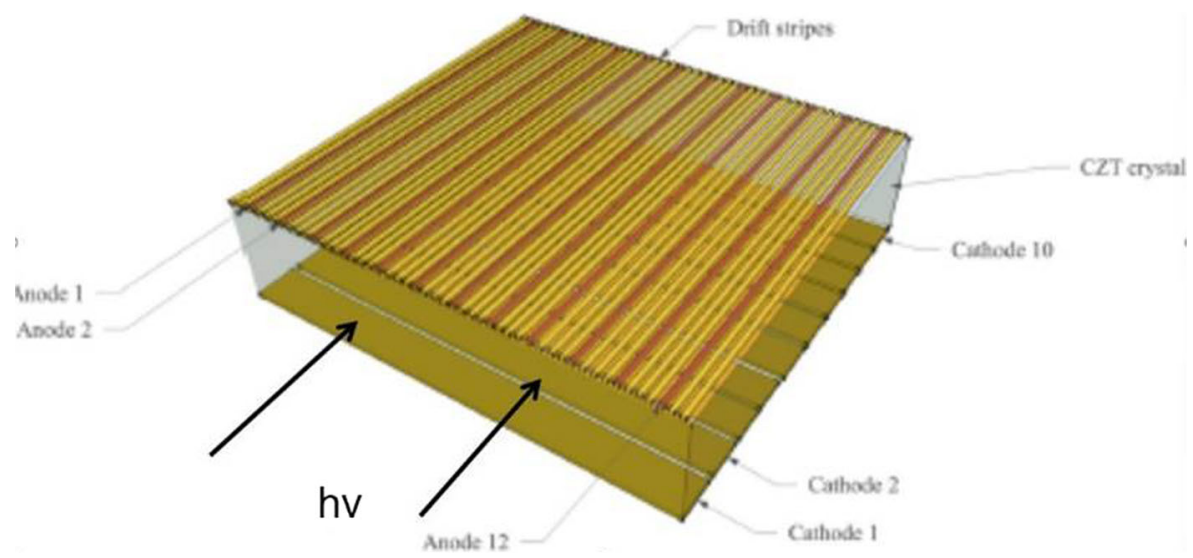

Fig. 14 Strip configuration of a single detection element. The electric field is orthogonal to the incident photon direction 


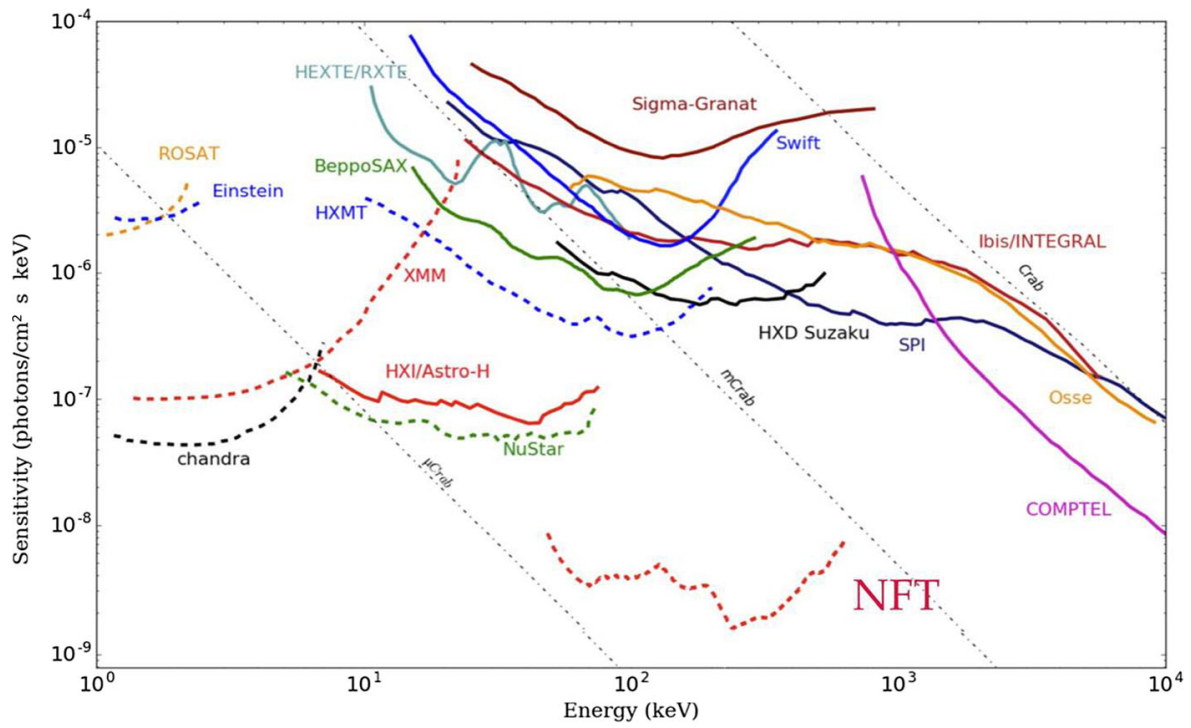

Fig. 15 NFT continuum sensitivity at $3 \sigma$, with $\Delta E=E / 2$ and $\Delta \mathrm{T}=10^{5} \mathrm{~s}$

which is required to achieve, with a proper coded mask configuration (see below), a Point Source Localization Accuracy (PSLA) of 1 arcmin at low energies ( $<30 \mathrm{keV})$. The hexagonal cross section of the bars is crucial to obtain the instrument's unique polarimetric capabilities (see below). In the current design the scintillator material is $\mathrm{CsI}(\mathrm{Tl})$, but also other materials (like BGO and GAGG(Ce)) will be considered.

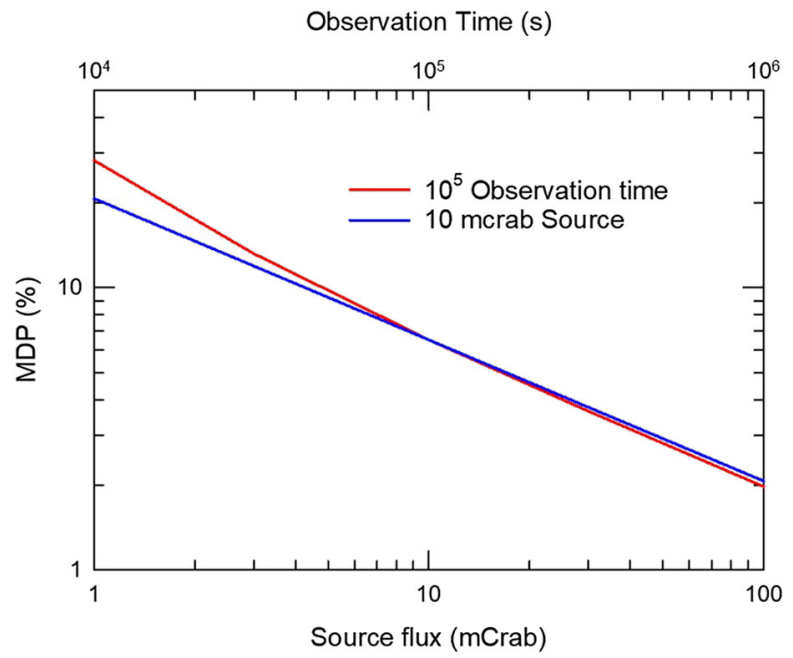

Fig. 16 Minimum Detectable Polarization (MDP) in $10^{5} \mathrm{~s}$ as a function of the polarized source intensity (red line) and for a $10 \mathrm{mCrab}$ source as a function of the observation time (blue line). In both cases, we assumed a modulation factor of the detector $Q_{100}=0.6$ 


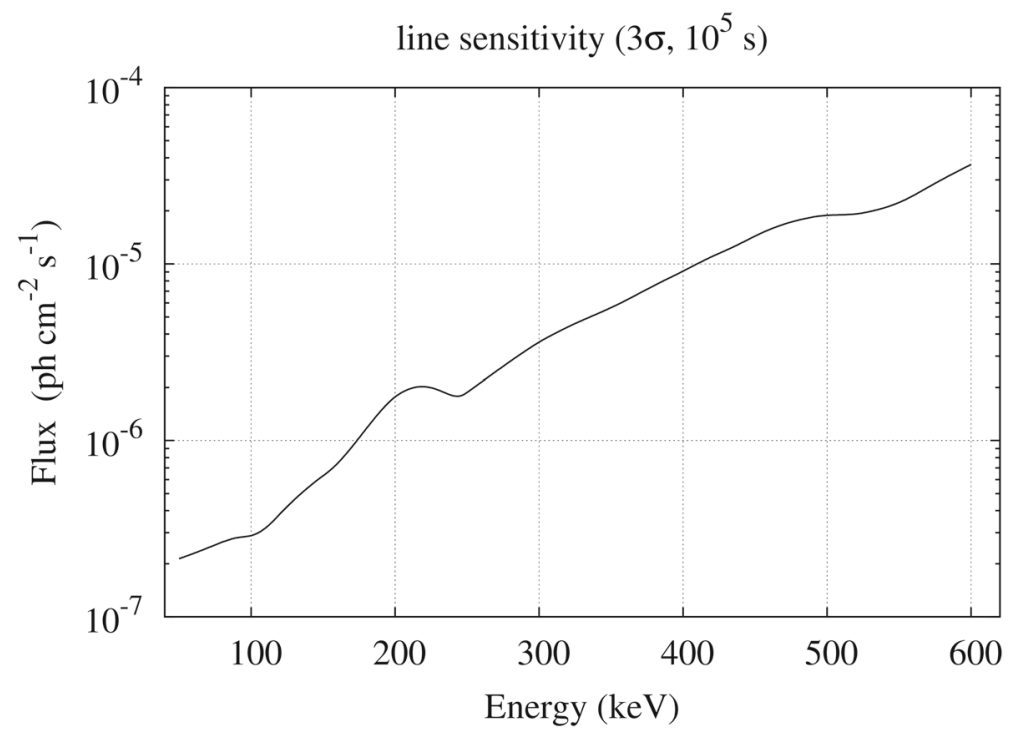

Fig. 17 Expected line sensitivity for the NFT on board ASTENA, calculated for an observation time of $10^{5} \mathrm{~s}$, at $3 \sigma$ confidence level

Depending on the scintillator material, the length of the bars will be optimized also for exploiting the Compton interactions in different bars for the determination, under study, of the incident photon direction. Preliminary results, that will be part of a paper in preparation, have already been obtained for $\mathrm{CsI}(\mathrm{Tl})$ bars $50 \mathrm{~mm}$ long. The reported performance estimates are based on the assumption that the scintillation bars are made of $\mathrm{CsI}(\mathrm{Tl}) 50 \mathrm{~mm}$ long (see Fig. 8).

\subsubsection{Coded mask}

Imaging capabilities of the WFM-IS are obtained by means of a double scale coded mask, one scale for the high energy photons $(30-150 \mathrm{keV})$ that lose their energy in the scintillator bars, and another scale for the low energy photons $(<30 \mathrm{keV})$ that lose their energy in the SDD alone. The high energy mask is made of Tungsten $1 \mathrm{~mm}$ thick, while the low energy mask is made of stainless steel $0.5 \mathrm{~mm}$ thick. The high energy mask is a $43 \times 41$ element basic pattern of $10 \times 10.1 \mathrm{~mm}$, with $79 \times 79$ of such elements for each detection unit, with $50 \%$ of open 2-dimension (2D) pixels, a Fully Coded Field of View (FCFOV) of 0.27 sr, and a Full Width at Zero Response (FWZR) of 2 sr. The expected Point Source Location Accuracy (PSLA) is approximately 5 arcmin for a $7 \sigma$ signal, and better for higher $\mathrm{S} / \mathrm{N}$ signals.

Unlike the high energy mask, the low energy mask is 1-D (see left panel of Fig. 9), with a throughput of $\sim 65 \%$, so as to allow the combined 2D/1D mask pattern (see right panel of Fig. 9) to have a throughput of 50\% at high energies and about 33\% at low energies. With these properties, by orienting the 1-D mask of each pair of detectors (see Fig. 5) perpendicularly to each other, we can achieve a PSLA of 1 arcmin, 
which is required to perform NFT follow-up observations of sources discovered with the WFM-IS.

\subsubsection{WFM-IS polarimetric capabilities}

Thanks to the hexagonal cross section of the scintillator bars and the 3D position sensitivity of the detector, each detection unit can be used as a Compton scattering polarimeter. In Fig. 10, we show the Minimum Detectable Polarization at $3 \sigma$ level in the case of a GRB of $20 \mathrm{~s}$ duration and 2 different fluences. The assumed spectrum is a Band law with low-energy photon index $\alpha=1.0$, high energy photon index $\beta=2.3$, and peak energy of the $E F(E)$ spectrum equal to $300 \mathrm{keV}$.

\subsubsection{WFM-IS continuum sensitivity}

Considering the throughput of the coded mask, the geometric area of the WFM-IS through the mask is $\sim 5800 \mathrm{~cm}^{2}$ below $30 \mathrm{keV}$ and $\sim 6700 \mathrm{~cm}^{2}$ in $30-150 \mathrm{keV}$. At higher energies the mask is transparent to the radiation therefore the on-axis area is about $1.4 \mathrm{~m}^{2}$. Assuming the background level expected for a nearly equatorial orbit, Fig. 11 shows the WFM-IS continuum sensitivity as a function of the exposure time for a source in the FOV of the NFT.

\subsection{Narrow Field Telescope, NFT}

As mentioned above, the NFT is based on a broad band (50-600 keV) Laue lens with $\sim 3 \mathrm{~m}$ diameter and $20 \mathrm{~m}$ focal length (see sketch in the left panel of Fig. 12), with a Position Sensitive Detector (PSD) in the focal plane. We separately discuss both, Laue lens and PSD.

\subsubsection{Laue lens}

For a review on the physics underlying the Laue lenses see [30]. Briefly, Laue lenses exploit the crystal diffraction in transmission configuration (Laue geometry). A Laue lens is made of a large number of crystal tiles in transmission configuration, that are disposed in such a way that they concentrate the incident radiation onto a common focal spot. A convenient way to visualize the geometry of a crystal lens is to consider it as a spherical cup covered with rings of crystal tiles having their diffracting planes perpendicular to the sphere and the focal spot is on the symmetry axis at a focal distance $f=R / 2$ from the cup, with $\mathrm{R}$ being the curvature radius of the spherical cup. From the Bragg law and the geometry of the lens, it can be easily demonstrated that the reflected energy from a crystal at distance $r$ from the lens symmetry axis is given by [30]

$$
E=\frac{h c}{2 d_{h k l}} \sin \left[\frac{1}{2} \arctan \left(\frac{f}{r}\right)\right] \approx \frac{h c f}{d_{h k l} r}
$$


where $d_{h k l}$ (in $\AA$ ) is the distance between the chosen lattice planes ( $h k l$ Miller indices) of the crystals, $h c=12.4 \mathrm{keV} \cdot \AA$ and $E$ is the energy of the gamma-ray photon (in $\mathrm{keV}$ ). The approximated expression is valid for gamma-ray lenses, given the small diffraction angles involved. Thus the highest energies are diffracted from the nearest crystals and the lowest energies from the furthest crystals to the lens axis. At the University of Ferrara, with the project HAXTEL (Hard X-ray TELescope) we developed a technology for assembling Laue lenses with moderate focal length $(<$ $10 \mathrm{~m}$ ), using flat mosaic crystal tiles of Copper $\mathrm{Cu}(111)$ [32, 78].

The disadvantage of flat crystals is that the minimum focal spot size is that of the crystal tiles. To overcome this limitation, bent crystals with the proper curvature are required. To this end, a technology for bending crystals and for assembling a Laue lens with long focal length $(20 \mathrm{~m})$ for astrophysical applications, was successfully developed as part of the LAUE project supported by the Italian Space Agency (ASI) (see, e.g, [80]). With the support of the European project AHEAD (integrated Activities in the High Energy Astrophysics Domain), the expected performance of a Laue lens prototype has also been simulated taking into account effects of possible crystal tile misalignments and radial distortions of the crystal curvature, as measured in laboratory experiments.

The lens under development is made of $\sim 19500$ bent crystal tiles of $\mathrm{Si}(111)$ and $\mathrm{Ge}(111$ ), with $40 \mathrm{~m}$ curvature radius (within 5\% uncertainty). In the current design the crystal tiles have a $30 \times 10 \mathrm{~mm}^{2}$ cross section, and a $2 \mathrm{~mm}$ thickness. Bent crystals have been produced and a technology for an accurate alignment (the requirement is a misalignment $<10$ arcsec) of the crystal tiles in the lens has been identified [79] with successful results (paper in preparation). The development of a lens prototype is the goal of a recently approved project TRILL (Technological Readiness level Increase for Laue Lenses) devoted to increase the Technology Readiness Level (TRL). The Laue lens we propose for ASTENA builds on this recent technological progress. The expected Point Spread Function of the entire lens, validated by measurements on several subsets of crystals, is shown in the right panel of Fig 12, with a Half Energy Width (HEW) of 30 arcsec.

Table 1 Main properties of the WFM-IS and of the NFT on board ASTENA

\begin{tabular}{lll}
\hline & WFM-IS & NFT \\
\hline Energy pass-band & $2 \mathrm{keV}-20 \mathrm{MeV}$ & $50-600 \mathrm{keV}$ \\
Total useful area $^{(a)}$ & $\sim 5800 \mathrm{~cm}^{2}(<30 \mathrm{keV})$ & \\
& $\sim 6700 \mathrm{~cm}^{2}(30-150 \mathrm{keV})$ & $7 \mathrm{~m}^{2}$ (projected) \\
& $\sim 13800 \mathrm{~cm}^{2}(>200 \mathrm{keV})$ & \\
Field of View & $2 \mathrm{sr}$ & $\sim$ arcmin \\
Angular resolution & $6 \operatorname{arcmin}$ & $<10$ arcsec HPD \\
Point source localization accuracy & $1 \operatorname{arcmin}($ see text) \\
Minimum Detectable Polarization & see Fig. 10 & see Fig. 16 \\
\hline
\end{tabular}

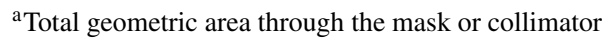


Important features of the proposed lens are its energy passband, angular resolution, and Field of View (FOV). Thanks to the use of bent $\mathrm{Si}(111)$ and $\mathrm{Ge}(111)$ crystals tiles, an energy passband 50-600 keV is guaranteed; however we will explore the possibility to extend the energy band down to $30 \mathrm{keV}$, if the transparency of the passive materials in the lens under the crystals is still acceptable. Using simulations, validated by laboratory measurements on the performance of a large sample of bent crystals, we evaluated the angular resolution and FOV of the proposed lens. As shown in Fig. 13, an angular resolution of 0.5 arcmin can be achieved, with a useful FOV of approximately 4 arcmin.

\subsubsection{Focal plane detector}

The focal-plane detector is a solid state PSD made of 4 layers, with each layer made of $4 \times 16 \mathrm{CdZnTe}(\mathrm{CZT})$ elements. Each element is $2 \mathrm{~cm}$ thick with a cross section of $0.5 \times 2 \mathrm{~cm}^{2}$, which exhibits a drift strip configuration for the anodes and orthogonally segmented cathodes in Parallel Transverse Field (PTF; see Fig. 14, [5, 51]). The resulting PSD has a total cross section of $8 \times 8 \mathrm{~cm}^{2}$ and a total thickness of $8 \mathrm{~cm}$. With this thickness, the PSD has a detection efficiency higher than $80 \%$ in the entire energy band of the NFT. The measured three-dimensional position resolution of a single element matches that required by the ASTENA NFT: $300 \mu \mathrm{m}[50,51]$. In this configuration, the PSD is also capable to work as a Compton polarimeter [16].

\subsubsection{Expected NFT performance}

\section{- Sensitivity to continuum emission}

The expected NFT continuum sensitivity $\left(3 \sigma, 10^{5} \mathrm{~s}, \Delta \mathrm{E}=E / 2\right)$ is shown in Fig. 15, compared with that of other missions or experiments. This unprecedented sensitivity (up to two orders of magnitude better than operating instruments in the same energy band) is obtained thanks mainly to the use of properly bent crystals and to the transmission geometry, that allows a projected geometric area of about $7 \mathrm{~m}^{2}$, with a very low mass of the lens $(\sim 150 \mathrm{~kg})$.

\section{- Sensitivity to polarized radiation}

The unprecedented sensitivity, together with the focal plane characteristics (high segmentation, spatial resolution in $3 \mathrm{D}$, and good energy resolution) will allow the NFT to enter a completely new territory in high-energy polarimetric measurements, making polarimetry a standard observation mode between $100 \mathrm{keV}$ and 600/700 keV [15]. To measure the reliability of polarimetric measurements, we adopt the Minimum Detectable Polarization (MDP) parameter, which quantifies the confidence with which polarization is detected, i.e. that the source is not un-polarized. MDP should be significantly smaller than the degree of polarization to be measured. Following [82], the MDP at $99 \%$ confidence level can be expressed by

$$
M D P_{99 \%}=\frac{4.29}{A \epsilon S_{F} Q_{100}} \sqrt{\frac{A \epsilon S_{F}+B}{T}}
$$


where, in the case of a focusing instrument, $A$ is the effective area of the focusing telescope, $\epsilon$ is the detection efficiency (i.e., the double events efficiency for a scattering polarimeter), $S_{F}$ is the source flux over the selected energy band (photons $\mathrm{cm}^{-2} \mathrm{~s}^{-1}$ ), $B$ is the background count rate (counts/s) integrated inside the volume of the detector subtended by the lens PSF, and $T$ is the observation time. The factor $Q_{100}$ is the polarimetric modulation factor of the detector (i.e., the measured modulation for a source $100 \%$ linearly polarized). The MDP achievable by the NFT can be estimated by evaluating the source count rate from the expected sensitivity reported in Fig. 15, in the energy band between $80 \mathrm{keV}$ and $600 \mathrm{keV}$, and setting $Q_{100}=0.6$. The latter is a realistic value for the NFT required focal plane, as can be inferred from different experiments performed using CdTe spectro-imagers operated as scattering polarimeters [4, 24]. Figure 16 shows this estimate as a function of both the observing time for a 10 $\mathrm{mCrab}$ source, and the source intensity integrated over $10^{5} \mathrm{~s}$. A Crab-like spectrum is assumed, with a background level given by [26] and later confirmed with the INTEGRAL SPI instrument, scaled to a low-Earth orbit. These results confirm unprecedented polarimetric performance of the NFT telescope with an achievable MDP of $5 \%$ for a $10 \mathrm{mCrab}$ source for a $10^{5} \mathrm{~s}$ observation time.

\section{- Sensitivity to narrow emission lines}

In general, for a focusing telescope, the sensitivity to a narrow emission line expressed in photons $\mathrm{cm}^{-2} \mathrm{~s}^{-1}$, superimposed onto a continuum source spectrum, at a confidence level of $n_{\sigma}$, is given by:

$$
I_{L}^{\text {min }}\left(E_{L}\right)=1.31 n_{\sigma} \frac{\sqrt{\left[2 B\left(E_{L}\right) A_{d}+I_{c}\left(E_{L}\right) \eta_{d} f_{\epsilon} A_{e f f}\right] \Delta E}}{\eta_{d} f_{\epsilon} A_{e f f} \sqrt{T_{o b s}}}
$$

where $\mathrm{E}_{L}$ is the line centroid, $I_{c}\left(E_{L}\right)$ is the source continuum intensity at the line centroid, $\triangle \mathrm{E}$ is the FWHM of the line profile that depends upon the energy resolution of the detector which has been assumed to be $2 \mathrm{keV}$ (expected for our simulated detector; [46]). The other parameters, all calculated at the energy $E_{L}$, are those defined above.

The sensitivity to an emission line for our Laue lens, at $3 \sigma$ confidence level, is shown in Fig. 17 for an observation time of $10^{5} \mathrm{~s}$. Here we have adopted a typical scatter of the radial curvature of bent crystals (Gaussian deviation of $2.5 \%$ from the mean) and a tile misalignment within 10 arcsec; a continuum intensity given by our continuum sensitivity, a FWHM of the line of $2 \mathrm{keV}$ and the fraction $f_{\epsilon}=0.5$, corresponding to a half energy width of the PSF. This figure shows how the line sensitivity of the ASTENA/NFT in $10^{5} \mathrm{~s}$ is approximately three orders of magnitude better than the SPI instrument aboard INTEGRAL at low energy, while it is about one order of magnitude better at $511 \mathrm{keV}^{3}$ This sensitivity can be further improved with a suitable choice and configuration of the reflecting planes of bent crystals in the lens.

In the common pass-band, a comparison of these performances with the NASA Medium Probe design mission AMEGO [58] shows that ASTENA has a

\footnotetext{
${ }^{3}$ https://www.cosmos.esa.int/web/integral/observation-time-estimator, see also [70]
} 
much higher sensitivity at low energies, while at $511 \mathrm{keV}$ ASTENA's sensitivity is similar, but with an order of magnitude better angular resolution.

\section{Conclusions}

The ESA Science Programme "Voyage 2050" offers a formidable opportunity to address issues of primary importance for astrophysics and, more generally, for science. In this WP we have discussed two relevant open problems: 1) a detailed understanding of the physical processes that underpin the explosions of Type-Ia and core collapse supernovae; 2) the fifty-year long mystery of the origin of the $511 \mathrm{keV}$ positron annihilation line from the Galactic bulge region. Their solution has been thus far hindered by the limited sensitivity and imaging capabilities of the gamma ray instrumentation flown and could be solved with the proposed satellite mission concept ASTENA. Thanks to its broad band (2 keV-20 MeV) WFM-IS and its focusing NFT with a 50-600 keV passband, ASTENA will provide hitherto unparalleled sensitivity to continuum and line emission, polarimetric capabilities, and unprecedented sub-arcmin angular resolution, that will make it a first-class investigation tool for high energy astrophysics, and will make a breakthrough possible in this field in general, and in the above poorly known areas in particular. Besides settling the problems we have discussed in this paper, ASTENA can tackle many other issues, like those concerning the transient sky (see WP by [39]), and several classes of Galactic (e.g., magnetars) and extragalactic (e.g., Blazars) sources, thus far scarcely explored at hard $\mathrm{X} /$ soft gamma rays (paper in preparation).

Acknowledgements The ASTENA mission concept is the result of several development activities. We wish to acknowledge the support by the Italian Space Agency (ASI) and that by the AHEAD European program.

Funding Open access funding provided by Università degli Studi di Ferrara within the CRUI-CARE Agreement.

Open Access This article is licensed under a Creative Commons Attribution 4.0 International License, which permits use, sharing, adaptation, distribution and reproduction in any medium or format, as long as you give appropriate credit to the original author(s) and the source, provide a link to the Creative Commons licence, and indicate if changes were made. The images or other third party material in this article are included in the article's Creative Commons licence, unless indicated otherwise in a credit line to the material. If material is not included in the article's Creative Commons licence and your intended use is not permitted by statutory regulation or exceeds the permitted use, you will need to obtain permission directly from the copyright holder. To view a copy of this licence, visit http://creativecommons.org/licenses/by/4.0/.

\section{References}

1. Ahmad, I., Greene, J.P., Moore, E.F., Ghelberg, S., Ofan, A., Paul, M., Kutschera, W.: Improved measurement of the Ti44 half-life from a 14-year long study. Phys. Rev. C 74(6), 065803 (2006). https://doi.org/10.1103/PhysRevC.74.065803 
2. Albernhe, F., Le Borgne, J.F., Vedrenne, G., Boclet, D., Durouchoux, P., da Costa, J.M.: Detection of the positron annihilation gamma ray line from the Galactic Center region. A \& A 94(2), 214-218 (Feb 1981)

3. Alexis, A., Jean, P., Martin, P., Ferrière, K.: Monte Carlo modelling of the propagation and annihilation of nucleosynthesis positrons in the Galaxy. A \& A 564, A108 (2014)

4. Antier, S., Ferrando, P., Limousin, O., Caroli, E., Curado da Silva, R.M., et al.: Hard X-ray polarimetry with Caliste, a high performance CdTe based imaging spectrometer. Exp. Astron. 39, 233-258 (2015). https://doi.org/10.1007/s10686-015-9442-5

5. Auricchio, N., Caroli, E., Basili, A., et al.: Development status of a CZT spectrometer prototype with 3D spatial resolution for hard x-ray astronomy. In: High Energy, Optical, and Infrared Detectors for Astronomy V, volume 8453 of Proc. SPIE, p. 84530S (2012). https://doi.org/10.1117/12.928327

6. Bandyopadhyay, R.M., Silk, J., Taylor, J.E., Maccarone, T.J.: On the origin of the 511-keV emission in the Galactic Centre. MNRAS 392, 1115-1123 (2009)

7. Bartels, R., Calore, F., Storm, E., Weniger, C.: Galactic binaries can explain the Fermi Galactic centre excess and $511 \mathrm{keV}$ emission. arXiv.org 480, 3826-3841 (2018)

8. Bisnovatyi-Kogan, G.S., Pozanenko, A.S.: Can flare stars explain the annihilation line from the galactic bulge? Astrophysics 60(2), 223-227 (2017)

9. Bœhm, C.: The dark matter interpretation of the $511 \mathrm{keV}$ line. N. J. Phys. 11(10), 105009 (2009). https://doi.org/10.1088/1367-2630/11/10/105009

10. Boehm, C., Hooper, D., Silk, J., Casse, M., Paul, J.: Mev dark matter: Has it been detected? Phys. Rev. Lett. 101301, 92 (2004). https://doi.org/10.1103/PhysRevLett.92.101301

11. Boggs, S.E., Harrison, F.A., Miyasaka, H., Grefenstette, B.W., et al.: ${ }^{44}$ Ti gamma-ray emission lines from SN1987A reveal an asymmetric explosion. Science 348(6235), 670-671 (2015). https://doi.org/10.1126/science.aaa2259

12. Bouchet, L., Mandrou, P., Roques, J.P., Vedrenne, G., et al.: SIGMA discovery of variable, E +- E - annihilation radiation from the near galactic center variable compact source 1E 1740.7-2942. ApJ1 383, L45 (1991). https://doi.org/10.1086/186237

13. Bouchet, L., Strong, A., Jourdain, E., Roques, J.P., Porter, T., Moskalenko, I., Diehl, R., Orlando, E.: A complete all-sky survey with INTEGRAL/SPI: sources census, hard X-ray diffuse emission and annihilation line. In: The Extreme Sky: Sampling the Universe above $10 \mathrm{keV}$, page 16 (2009)

14. Campana, R., Fuschino, F., Labanti, C., Amati, L., Mereghetti, S., Fiorini, M., Frontera, F., Baldazzi, G., Bellutti, P., et al.: The X-Gamma imaging spectrometer (XGIS) onboard THESEUS. Mem. Soc. Astron. Italiana 89, 137 (2018)

15. Caroli, E., Curado da Silva, R.M., Stephen, J.B., Pisa, A., Auricchio, N., Del Sordo, S., Donati, A., Schiavone, F., Landini, G., Honkimaki, V., Frontera, F.: A Polarimetric Experiment With a Laue Lens and CZT Pixel Detector. IEEE Trans. Nucl. Sci. 56(4), 1848-1854 (2009). https://doi.org/10.1109/TNS.2009.2021475

16. Caroli, E., Moita, M., da Silva, R., Del Sordo, S., de Cesare, G., Maia, J., Pàscoa, M.: Hard X-ray and soft gamma ray polarimetry with CdTe/CZT spectro-imager. Galaxies 6, 69 (2018). https://doi.org/10.3390/galaxies6030069

17. Cavallari, E., Frontera, F.: Hard X-ray/soft gamma-ray experiments and missions: overview and prospects. Space Sci. Rev. 212, 429-518 (2017). https://doi.org/10.1007/s11214-017-0426-9

18. Celotti, A., Blandford, R.D.: On the formation of jets. In: Kaper, L., Heuvel, E.P.J.V.D., Woudt, P.A. (eds.) Black Holes in Binaries and Galactic Nuclei, p. 206 (2001)

19. Chan, M.H., Leung, C.H.: Constraining dark matter by the $511 \mathrm{keV}$ line. MNRAS 479(2), 2229-2234 (2018). https://doi.org/10.1093/mnras/sty1583

20. Churazov, E., Sunyaev, R., Sazonov, S., Revnivtsev, M., Varshalovich, D.: Positron annihilation spectrum from the Galactic Centre region observed by SPI/INTEGRAL. MNRAS 357(4), 1377-1386 (2005). https://doi.org/10.1111/j.1365-2966.2005.08757.x

21. Churazov, E., Sunyaev, R., Isern, J., Knödlseder, J., Jean, P., Lebrun, F., Chugai, N., Grebenev, S., Bravo, E., Sazonov, S., Renaud, M.: Cobalt-56 $\gamma$-ray emission lines from the type Ia supernova $2014 J$. Nature 512, 406-408 (2014). https://doi.org/10.1038/nature13672

22. Clayton, D.D., Hoyle, F.: Gamma-ray lines from novae. ApJl 187, L101 (1974). https://doi.org/10.1086/181406

23. Clayton, D.D., Colgate, S.A., Fishman, G.J.: Gamma-ray lines from young supernova remnants. ApJ 155, 75 (1969). https://doi.org/10.1086/149849 
24. Curado da Silva, R.M., Caroli, E., Stephen, J.B., et al.: Polarimetric performance of a Laue lens gamma-ray CdZnTe focal plane prototype. J. Appl. Phys. 104(8), 084903-084903 (2008). https://doi.org/10.1063/1.3000092

25. Daugherty, J.K., Harding, A.K.: Pair production in superstrong magnetic fields. Apj 273, 761-773 (1983). https://doi.org/10.1086/161411

26. Dean, A.J., Fan, L., Byard, K., Goldwurm, A., Hall, C.J.: The gamma-ray emissivity of the earth's atmosphere. A \& A 219, 358-361 (1989)

27. Diehl, R.: Gamma rays from a supernova of type Ia: SN2014J. Astronomische Nachrichten 336(5), 464 (Jun 2015). https://doi.org/10.1002/asna.201512179

28. Diehl, R., Siegert, T., Hillebrandt, W., et al.: Early ${ }^{56} \mathrm{Ni}$ decay gamma rays from SN2014J suggest an unusual explosion. Science 345(6201), 1162-1165 (2014). https://doi.org/10.1126/science.1254738

29. Finkbeiner, D.P., Weiner, N.: Exciting dark matter and the integral/spi $511 \mathrm{kev}$ signal. Phys. Rev. D 76, 083519 (2007). https://doi.org/10.1103/PhysRevD.76.083519

30. Frontera, F., von Ballmoos, P.: Laue Gamma-Ray lenses for space astrophysics: status and prospects. X-Ray Optics and Instrumentation, 2010, Special Issue on X-Ray Focusing: Techniques and Applications, id.215375, 2010:215375, 2010. https://doi.org/10.1155/2010/215375

31. Frontera, F., Costa, E., dal Fiume, D., Feroci, M., Nicastro, L., Orlandini, M., Palazzi, E., Zavattini, G.: The high energy instrument PDS on-board the BeppoSAX X-ray astronomy satellite. Astron. Astrophys. Suppl. Series 122, 357-369 (1997). https://doi.org/10.1051/aas:1997140

32. Frontera, F., Loffredo, G., Pisa, A., Nobili, F., Carassiti, V., Evangelisti, F., Landi, L., Squerzanti, S., Caroli, E., Stephen, J.B., Andersen, K.H., Courtois, P., Auricchio, N., Milani, L., Negri, B.: Focusing of gamma-rays with Laue lenses: first results. In: Society of Photo-Optical Instrumentation Engineers (SPIE) Conference Series, vol. 7011 (2008)

33. Gehrels, N., Barthelmy, S.D., Teegarden, B.J., Tueller, J., Leventhal, M., MacCallum, C.J.: GRIS observations of positron annihilation radiation from the Galactic center. ApJ1 375, L13-L16 (1991). https://doi.org/10.1086/186077

34. Goldwurm, A., Ballet, J., Cordier, B., Paul, J., Bouchet, L., Roques, J.P., Barret, D., Mandrou, P., Sunyaev, R., Churazov, E., Gilfanov, M., Dyachkov, A., Khavenson, N., Kovtunenko, V., Kremnev, R., Sukhanov, K.: Sigma/GRANAT soft gamma-ray observations of the X-ray nova in Musca - Discovery of positron annihilation emission line. Astrophys. J. Lett. 389, L79-L82 (1992)

35. Gómez-Gomar, J., Isern, J., Jean, P.: Prospects for Type IA supernova explosion mechanism identification with gamma rays. MNRAS 295, 1-9 (1998). https://doi.org/10.1046/j.1365-8711.1998.29511115.x

36. Grebenev, S.A., Lutovinov, A.A., Tsygankov, S.S., Winkler, C.: Hard-X-ray emission lines from the decay of ${ }^{44} \mathrm{Ti}$ in the remnant of supernova 1987A. Nature 490, 373-375 (2012). https://doi.org/10.1038/nature11473

37. Grefenstette, B.W., Harrison, F.A., Boggs, S.E., Reynolds, S.P., Fryer, C.L., et al.: Asymmetries in core-collapse supernovae from maps of radioactive ${ }^{44} \mathrm{Ti}$ in CassiopeiaA. Nature 506, 339-342 (2014). https://doi.org/10.1038/nature12997

38. Grefenstette, B.W., Fryer, C.L., Harrison, F.A., Boggs, S.E., et al.: The Distribution of Radioactive ${ }^{44} \mathrm{Ti}$ in Cassiopeia A. ApJ 834, 19 (2017). https://doi.org/10.3847/1538-4357/834/1/19

39. Guidorzi, C., Frontera, F., Ghirlanda, G., Stratta, G., Mundell, C.G., Virgilli, E., Rosati, P., Caroli, E., Amati, L., Pian, E., Kobayashi, S., Ghisellini, G., Fryer, C., Della Valle, M., Margutti, R., Marongiu, M., Martone, R., Campana, R., Fuschino, F., Labanti, C., Orlandini, M., Stephen, J.B., Brandt, S., Curado da Silva, R.M., Laurent, R., abd Mochkovitch, P., Bozzo, E., Ciolfi, R., Burderi, L., Di Salvo, T.: A Deep Study of the High-Energy Transient Sky. Experimental Astronomy, this issue (2021)

40. Hailey, C.J., Mori, K., Bauer, F.E., Berkowitz, M.E., Hong, J., Hord, B.J.: A density cusp of quiescent X-ray binaries in the central parsec of the Galaxy. Nature 556, 70-73 (2018). https://doi.org/10.1038/nature25029

41. Harrison, F.A., Craig, W.W., Christensen, F.E., Hailey, C.J., Zhang, W.W., et al.: The nuclear spectroscopic telescope array (NuSTAR) high-energy X-Ray mission. Astrophys. J. 770, 103 (2013)

42. Hungerford, A., Fryer, C.: Astrophysical Radionuclides: Where are they and what can they tell us? In: Bulletin of the American Physical Society, vol. 64 (2019)

43. Isern, J., Jean, P., Bravo, E., Knödlseder, J., et al.: Gamma-ray emission from SN2014J near maximum optical light. A \& A 588, A67 (2016). https://doi.org/10.1051/0004-6361/201526941

44. Jean, P., Knödlseder, J., Gillard, W., et al.: Spectral analysis of the Galactic $\mathrm{e}^{+e^{-}}$annihilation emission. Astron. Astrophys. 445(2), 579-589 (2006) 
45. Johnson, W.N. III., Harnden, F.R. Jr.., Haymes, R.C.: The spectrum of low-energy gamma radiation from the Galactic-center region. ApJ1 172, L1 (1972). https://doi.org/10.1086/180878

46. Khalil, M., Frontera, F., Caroli, E., Virgilli, E., Valsan, V.: A simulation study on the focal plane detector of the LAUE project. Nucl. Inst. Methods Phys. Res. A 786, 59-70 (2015). https://doi.org/10.1016/j.nima.2015.03.004

47. Knödlseder, J., Jean, P., Lonjou, V., Weidenspointner, G., Guessoum, N., Gillard, W., Skinner, G., von Ballmoos, P., Vedrenne, G., Roques, J.-P., Schanne, S., Teegarden, B., Schönfelder, V., Winkler, C.: The all-sky distribution of $511 \mathrm{keV}$ electron-positron annihilation emission. A\&A 441, 513-532 (2005). https://doi.org/10.1051/0004-6361:20042063

48. Korobkin, O., Hungerford, A.M., Fryer, C.L., Mumpower, M.R., Misch, G.W., Sprouse, T.M., Lippuner, J., Surman, R., Couture, A.J., Bloser, P.F., Shirazi, F., Even, W.P., Vestrand, W.T., Miller, R.S.: Gamma-rays from kilonova:, a potential probe of r-process nucleosynthesis. arXiv:1905.05089 (2019)

49. Kuchner, M.J., Kirshner, R.P., Pinto, P.A., Leibundgut, B.: Evidence for Ni-56 yields Co-56 yields Fe-56 decay in type IA supernovae. ApJ1 426, 89-92 (1994). https://doi.org/10.1086/187347

50. Kuvvetli, I., Budtz-Jørgensen, C., Caroli, E., Auricchio, N.: CZT drift strip detectors for high energy astrophysics. Nucl. Instrum. Methods Phys. Res. A 624, 486-491 (2010). https://doi.org/10.1016/j.nima.2010.03.172

51. Kuvvetli, I., Budtz-Jørgensen, C., Zappettini, A., et al.: A 3D CZT high resolution detector for xand gamma-ray astronomy. In: High energy, optical, and infrared detectors for astronomy VI, volume 9154 of Proc. SPIE, pp. $91540 X$ (2014). https://doi.org/10.1117/12.2055119

52. Leventhal, M., MacCallum, C.J., Stang, P.D.: Detection of $511 \mathrm{keV}$ positron annihilation radiation from the galactic center direction. ApJl 225, L11-L14 (1978). https://doi.org/10.1086/182782

53. Leventhal, M., MacCallum, C.J., Huters, A.F., Stang, P.D.: Gamma-ray lines and continuum radiation from the galactic center direction. ApJ 240, 338-343 (1980). https://doi.org/10.1086/158237

54. Leventhal, M., MacCallum, C.J., Huters, A.F., Stang, P.D.: Current status of the galactic center positron-annihilation source. ApJ 302, 459-461 (1986). https://doi.org/10.1086/164004

55. Lingenfelter, R.E., Ramaty, R.: The nature of the annihilation radiation and gamma-ray continuum from the galactic center region. ApJ 343, 686 (1989). https://doi.org/10.1086/167740

56. Marisaldi, M., Labanti, C., Soltau, H.: A pulse shape discrimination Gamma-Ray detector based on a silicon drift chamber coupled to a $\mathrm{CsI}(\mathrm{Tl})$ scintillator: prospects for a $1 \mathrm{keV} 1 \mathrm{MeV}$ Monolithic detector. IEEE Trans. Nucl. Sci. 51, 1916-1922 (2004). https://doi.org/10.1109/TNS.2004.832679

57. Marisaldi, M., Labanti, C., Soltau, H., Fiorini, C., Longoni, A., Perotti, F.: X- and Gamma-Ray detection with a silicon drift detector coupled to a CsI(Tl) scintillator operated with pulse shape discrimination technique. IEEE Trans. Nucl. Sci. 52, 1842-1848 (2005). https://doi.org/10.1109/TNS.2005.856615

58. McEnery, J., van der Horst, A., Dominguez, A., Moiseev, A., Marcowith, A.r., Harding, A., Lien, A., Giuliani, A., Inglis, A., Ansoldi, S., Stamerra, A., Manousakis, A., Strong, A., Bambi, C., Patricelli, B., Baring, M., Barrio, J.A., Bastieri, D., Fields, B., Beacom, J., Beckmann, V., Bednarek, W., Rani, B., Boggs, S., Bolotnikov, A., Cenko, S.B., Buckley, J., Grefenstette, B., Hui, M., Pittori, C., PrescodWeinstein, C., Shrader, C., Gouiffes, C., Kierans, C., Wilson-Hodge, C., D’Ammando, F., Castro, D., Kocveski, D., Gasparrini, D., Thompson, D., Williams, D., De Angelis, A., Bernard, D., Digel, S., Morcuende, D., Charles, E., Bissaldi, E., Hays, E., Ferrara, E., Bozzo, E., Grove, E., Wulf, E., Bottacini, E., Caroli, E., Kislat, F., Oikonomou, F., Giordano, F., Longo, F., Fryer, C., Fukazawa, Y., Georganopoulos, M., De Nolfo, G., Vianello, G., Kanbach, G., Younes, G., Blumer, H., Hartmann, D., Hernanz, M., Takahashi, H., Li, H., Agudo, I., Moskalenko, I., Stumke, I., Grenier, I., Smith, J., Rodi, J., Perkins, J., Gelfand, J., Holder, J., Knodlseder, J., Kopp, J., Lenain, J.-P., Álvarez, J.-M., Metcalfe, J., Krizmanic, J., Stephen, J.B., Hewitt, J., Mitchell, J., Harding, P., Tomsick, J., Racusin, J., Finke, J., Kargaltsev, O., Klimenko, A.V., Krawczynski, H., Smith, K., Kubo, H., Di Venere, L., Marcotulli, L., Lommler, J., Parker, L., Baldini, L., Foffano, L., Zampieri, L., Tibaldo, L., Petropoulou, M., Ajello, M., Meyer, M., López, M., McConnell, M., Boettcher, M., Cardillo, M., Martinez, M., Kerr, M., Mazziotta, M.N., McEnery, J., Di Mauro, M., Wood, M., Meyer, E., Briggs, M., De Becker, M., Lovellette, M., Doro, M., Sanchez-Conde, M.A., Moss, M., Mizuno, T., Ribó, M., Nakazawa, K., Neilson, N.K., Auricchio, N., Omodei, N., Oberlack, U., Ohno, M., Orland o, E., Otte, N., Coppi, P., Bloser, P., Zhang, H., Laurent, P., Pohl, M., Prand ini, E., Shawhan, P., Caputo, R., Campana, R., Rando, R., Woolf, R., Johnson, R., Mignani, R., Walter, R., Ojha, R., da Silva, R.C., Dietrich, S., Funk, S., Zane, S., Anton, S., Buson, S., Cutini, S., Saz Parkinson, P., Schirato, R., Griffin, S., Kaufmann, S., Stawarz, L., Ciprini, S., Del Sordo, S., Jones, S., Guiriec, S., Tajima, H., Cheung, T., The, L.S., Venters, T., Porter, T., Linden, T., Barres, U., Paliya, V.S., Bozhilov, V., Vestrand, T., Tatischeff, 
V., Chen, W., Wang, X., Tanaka, Y., Uhm, L., Zhang, B., Zimmer, S., Zoglauer, A., Wadiasingh, Z.: All-sky medium energy gamma-ray observatory: exploring the extreme Multimessenger Universe. In: BAAS, vol. 51, p. 245 (2019)

59. Milne, P.A., The, L.S., Leising, M.D.: Positron Escape from Type IA Supernovae. ApJs 124(2), 503526 (1999). https://doi.org/10.1086/313262

60. Mróz, P., Udalski, A., Poleski, R., Soszyński, I., Szymański, M.K., Pietrzyński, G., Wyrzykowski, Ł., Ulaczyk, K., Kozłowski, S., P. Pietrukowicz, Skowron, J.: OGLE atlas of classical Novae. I. Galactic bulge objects. ApJs 219(2), 26 (2015). https://doi.org/10.1088/0067-0049/219/2/26

61. Phillips, M.M.: The absolute magnitudes of Type IA supernovae. ApJ1 413, L105-L108 (1993). https://doi.org/10.1086/186970

62. Prantzos, N., Boehm, C., Bykov, A.M., Diehl, R., Ferrière, K., Guessoum, N., Jean, P., Knoedlseder, J., Marcowith, A., Moskalenko, I.V., Strong, A., Weidenspointner, G.: The 511 $\mathrm{keV}$ emission from positron annihilation in the Galaxy. Rev. Mod. Phys. 83, 1001-1056 (2011). https://doi.org/10.1103/RevModPhys.83.1001

63. Purcell, W.R., Grabelsky, D.A., Ulmer, M.P., Johnson, W.N., Kinzer, R.L., Kurfess, J.D., Strickman, M.S., Jung, G.V.: OSSE observations of Galactic $511 \mathrm{keV}$ positron annihilation radiation - Initial phase 1 results. ApJl 413, L85-L88 (1993). https://doi.org/10.1086/186965

64. Purcell, W.R., Cheng, L.-X., Dixon, D.D., Kinzer, R.L., Kurfess, J.D., Leventhal, M., Saunders, M.A., Skibo, J.G., Smith, D.M., Tueller, J.: OSSE mapping of galactic $511 \mathrm{keV}$ positron annihilation line emission. ApJ 491, 725-748 (1997)

65. Renaud, M., Vink, J., Decourchelle, A., Lebrun, F., et al.: The Signature of ${ }^{44} \mathrm{Ti}$ in Cassiopeia A revealed by IBIS/ISGRI on INTEGRAL. ApJ1 647, L41-L44 (2006). https://doi.org/10.1086/507300

66. Share, G.H., Leising, M.D., Messina, D.C., Purcell, W.R.: Limits on a variable source of $511 \mathrm{keV}$ annihilation radiation near the Galactic center. ApJ1 358, L45-L48 (1990). https://doi.org/10.1086/185776

67. Siegert, T., Diehl, R., Krause, M.G.H., Greiner, J.: Revisiting INTEGRAL/SPI observations of ${ }^{44} \mathrm{Ti}$ from Cassiopeia A. A \& A 579, A124 (2015). https://doi.org/10.1051/0004-6361/201525877

68. Siegert, T., Diehl, R., Greiner, J., Krause, M.G.H., Beloborodov, A.M., Bel, M.C., Guglielmetti, F., Rodriguez, J., Strong, A.W., Zhang, X.: Positron annihilation signatures associated with the outburst of the microquasar V404 Cygni. Nature 531, 341-343 (2016a). https://doi.org/10.1038/nature16978

69. Siegert, T., Diehl, R., Khachatryan, G., Krause, M.G.H., Guglielmetti, F., Greiner, J., Strong, A.W., Zhang, X.: Gamma-ray spectroscopy of positron annihilation in the Milky Way. Astron. Astrophys. 586, A84 (2016b)

70. Siegert, T., Diehl, R., Vincent, A.C., Guglielmetti, F., Krause, M.G.H., Boehm, C.: Search for 511 $\mathrm{keV}$ emission in satellite galaxies of the Milky Way with INTEGRAL/SPI. A \& A 595, A25 (2016c)

71. Siegert, T., Crocker, R.M., Diehl, R., Krause, M.G.H., Panther, F.H., Pleintinger, M.M.M., Weinberger, C.: Constraints on positron annihilation kinematics in the inner Galaxy. Astron. Astrophys. 627, A126 (2019)

72. Sim, S.A., Mazzali, P.A.: On the $\gamma$-ray emission of Type Ia supernovae. MNRAS 385, 1681-1690 (2008). https://doi.org/10.1111/j.1365-2966.2008.12600.x

73. Skinner, G., Diehl, R., Zhang, X., Bouchet, L., Jean, P.: The Galactic distribution of the $511 \mathrm{keV}$ $\mathrm{e}^{+} / \mathrm{e}^{-}$annihilation radiation. In: Proceedings of the 10th INTEGRAL Workshop: "A Synergistic View of the High-Energy Sky” (INTEGRAL 2014). 15-19 September 2014. Annapolis, MD, USA, p. 054 (2014). Published online at http://pos.sissa.it/cgi-bin/reader/conf.cgi?confid=228

74. Teegarden, B.J., Cline, T.L., Gehrels, N., Palmer, D., Ramaty, R., Seifert, H., Hurley, K.H., Landis, D.A., Madden, N.W., Malone, D., Pehl, R., Owens, A.: TGRS observation of the galactic center annihilation line. Apj Lett 463, L75 (1996). https://doi.org/10.1086/310061

75. Timmes, F., Fryer, C., Timmes, F., Hungerford, A.L., Couture, A., Adams, F., Aoki, W., Arcones, A., Arnett, D., Auchettl, K., et al.: Catching element formation in the act ; the case for a new MeV Gamma-ray mission: radionuclide astronomy in the 2020s. In: BAAS, vol. 51, p. 2 (2019)

76. Vedrenne, G., Roques, J.-P., Schönfelder, V., Mandrou, P., Lichti, G.G., et al.: SPI: The spectrometer aboard INTEGRAL. Astron. Astrophys. 411, L63-L70 (2003)

77. Vink, J., Laming, J.M., Kaastra, J.S., Bleeker, J.A.M., et al.: Detection of the 67.9 and $78.4 \mathrm{keV}$ lines associated with the radioactive decay of ${ }^{4} 4 \mathrm{Ti}$ in Cassiopeia A. ApJ1 560, L79-L82 (2001). https://doi.org/10.1086/324172 
78. Virgilli, E., Frontera, F., Valsan, V., Liccardo, V., Carassiti, V., Evangelisti, F., Squerzanti, S.: Laue lenses for hard $\mathrm{x}$-/soft $\gamma$-rays: new prototype results. In: Society of Photo-Optical Instrumentation Engineers (SPIE) Conference Series, volume 8147 of Proc. SPIE, p. $81471 B$ (2011). https://doi.org/10.1117/12.895233

79. Virgilli, E., Frontera, F., Rosati, P., Liccardo, V., Squerzanti, S., Carassiti, V., Caroli, E., Auricchio, N., Stephen, J.B.: Hard X-ray broad band Laue lenses (80-600 keV): building methods and performances Society of Photo-Optical Instrumentation Engineers (SPIE) Conference Series, volume 9603 of Proc. SPIE, page 960308 (2015). https://doi.org/10.1117/12.2190335

80. Virgilli, E., Valsan, V., Frontera, F., Caroli, E., Liccardo, V., B. Stephen, J.: Expected performances of a Laue lens made with bent crystals. J. Astron. Telesc. Instrum. Syst. 3(4), 044001 (2017). https://doi.org/10.1117/1.JATIS.3.4.044001

81. Weidenspointner, G., Skinner, G., Jean, P., Knödlseder, J., von Ballmoos, P., Bignami, G., Diehl, R., Strong, A.W., Cordier, B., Schanne, S., Winkler, C.: An asymmetric distribution of positrons in the Galactic disk revealed by $\gamma$,-rays. Nature 451, 159-162 (2008). https://doi.org/10.1038/nature06490

82. Weisskopf, M.C., Elsner, R.F., Hanna, D., Kaspi, V.M., O’Dell, S.L., Pavlov, G.G., Ramsey, B.D.: The prospects for X-ray polarimetry and its potential use for understanding neutron stars. In: Becker, W. (ed.) Neutron Stars and Pulsars, .Astrophysics and Space Science Library, vol. 357, pp. 589-619. Springer (2009). https://doi.org/10.1007/978-3-540-76965-1_22

83. Wilkinson, R.J., Vincent, A.C., BÅhm, C., McCabe, C.: Ruling out the light weakly interacting massive particle explanation of the Galactic $511 \mathrm{keV}$ line. Phys. Rev. D 94(10), 103525 (2016). https://doi.org/10.1103/PhysRevD.94.103525

84. Wu, M.-R., Banerjee, P., Metzger, B.D., Martínez-Pinedo, G., Aramaki, T., Burns, E., Hailey, C.J., Barnes, J., Karagiorgi, G.: Finding the remnants of the Milky Way's last neutron star mergers. ApJ 880(1), 23 (2019). https://doi.org/10.3847/1538-4357/ab2593

Publisher's note Springer Nature remains neutral with regard to jurisdictional claims in published maps and institutional affiliations. 


\section{Affiliations}

F. Frontera ${ }^{1,2,3}$ (D) $\cdot$ E. Virgilli ${ }^{1} \cdot$ C. Guidorzi ${ }^{1} \cdot$ P. Rosati ${ }^{1} \cdot$ R. Diehl ${ }^{4} \cdot$ T. Siegert $^{5}$. C. Fryer ${ }^{6}$ - L. Amati ${ }^{2}$ - N. Auricchio ${ }^{2}$. R. Campana ${ }^{2}$ - E. Caroli ${ }^{2}$. F. Fuschino ${ }^{2}$. C. Labanti ${ }^{2}$ - M. Orlandini ${ }^{2}$ - E. Pian ${ }^{2}$ - J. B. Stephen ${ }^{2}$ - S. Del Sordo ${ }^{7}$.

C. Budtz-Jorgensen ${ }^{8} \cdot$ I. Kuvvetli ${ }^{8}$. S. Brandt ${ }^{8}$. R. M. Curado da Silva9,10 . P. Laurent ${ }^{11} \cdot$ E. Bozzo ${ }^{12} \cdot$ P. Mazzali ${ }^{13} \cdot$ M. Della Valle ${ }^{3,14}$

1 Department of Physics and Earth Sciences, University of Ferrara Via Saragat 1, I-44122 Ferrara, Italy

2 Observatory of Astrophysics and Space Sciences, National Institute of Astrophysics (INAF), Via Gobetti 101, 40129 Bologna, Italy

3 International Center for Relativistic Astrophysics, Piazzale della Repubblica 2, I-65122 Pescara, Italy

4 Max Planck Institut für extraterrestrische Physik, Giessenbachstr.1, Postfach 1312, D-85741 Garching, Germany

5 Center for Astrophysics and Space Sciences, University of California, San Diego, 9500 Gilman Dr, La Jolla, CA 92093, USA

6 Los Alamos National Laboratory, P.O. Box 1663, Los Alamos, New Mexico 87545, USA

7 Institute of Space Astrophysics and Cosmic Physics, INAF, Via Ugo La Malfa 153, 90146, Palermo, Italy

8 National Space Institute, Technical University of Denmark (DTU), Anker Engelunds Vej 1, 2800, Kongens Lyngby, Denmark

9 LIP-Laboratório de Instrumentação e Física Experimental de Partículas, Coimbra, Portugal

10 Physics Department, University of Coimbra, Coimbra, Portugal

11 IRFU, CEA, Universite Paris-Saclay, F-91191 Gif-sur-Yvette, France

12 Department of Astronomy, University of Geneva, Chemin d'Ecogia 16, 1290 Versoix, Switzerland

13 Astrophysics Research Institute, Liverpool John Moores University, Liverpool, L3 5RF, UK

14 Capodimonte Astronomical Observatory, INAF-Napoli, Salita Moiariello 16, 80131, Napoli, Italy 